\title{
Conservation and evolution of the sporulation gene set in diverse members of the Firmicutes
}

\section{Michael Y. Galperin ${ }^{1^{*}}$, Natalya Yutin ${ }^{1}$, Yuri I. Wolf ${ }^{1}$, Roberto Vera Alvarez ${ }^{1}$, and Eugene V. Koonin ${ }^{1^{*}}$}

${ }^{1}$ National Center for Biotechnology Information, National Library of Medicine, National Institutes of Health, Bethesda, Maryland 20894, USA

For correspondence.

Tel.: 301-435-5910, E-mail: galperin@ncbi.nlm.nih.gov;

Tel.: 301-435-5913, E-mail: koonin@ncbi.nlm.nih.gov

Running title: Sporulation genes in diverse firmicutes

ORCID:

Michael Y. Galperin, 0000-0002-2265-5572

Natalya Yutin, 0000-0002-3633-5123

Yuri I. Wolf, $\underline{0000-0002-0247-8708}$

Roberto Vera Alvarez, 0000-0002-4108-5982

Eugene V. Koonin, 0000-0003-3943-8299 


\section{ABSTRACT}

The current classification of the phylum Firmicutes (new name, Bacillota) features eight distinct classes, six of which include known spore-forming bacteria. In Bacillus subtilis, sporulation involves up to 500 genes, many of which do not have orthologs in other bacilli and/or clostridia. Previous studies identified about 60 sporulation genes of $B$. subtilis that were shared by all sporeforming members of the Firmicutes. These genes are referred to as the sporulation core or signature although many of these are found also in genomes of non-spore-formers. Using an expanded set of 180 firmicute genomes from 160 genera, including 76 spore-forming species, we investigated the conservation of the sporulation genes, in particular, seeking to identify lineages that lack some of the genes from the conserved sporulation core. The results of this analysis confirmed that many small acid-soluble spore proteins (SASPs), spore coat proteins, and germination proteins, which were previously characterized in bacilli, are missing in spore-forming members of Clostridia and other classes of Firmicutes. A particularly dramatic loss of sporulation genes was observed in the spore-forming members of the families Planococcaceae and Erysipelotrichaceae. Fifteen species from diverse lineages were found to carry skin (sigKinterrupting) elements of different sizes that all encoded SpoIVCA-like recombinases but did not share any other genes. Phylogenetic trees built from concatenated alignments of sporulation proteins and ribosomal proteins showed similar topology, indicating an early origin and subsequent vertical inheritance of the sporulation genes.

\section{IMPORTANCE}

Many members of the phylum Firmicutes (Bacillota) are capable of producing endospores, which enhance the survival of important Gram-positive pathogens that cause such diseases as anthrax, botulism, colitis, gas gangrene, and tetanus. We show that the core set of sporulation genes, defined previously through genome comparisons of several bacilli and clostridia, is conserved in a wide variety of spore-formers from several distinct lineages of Firmicutes. We also detect widespread loss of sporulation genes in many organisms, particularly within families Planococcaceae and Erysipelotrichaceae. Members of these families, such as Lysinobacillus sphaericus and Clostridium innocuum, could be excellent model organisms for studying sporulation mechanisms, such as engulfment, formation of the spore coat, and spore germination. 


\section{INTRODUCTION}

A variety of bacteria are capable of producing resting forms, commonly referred to as spores. However, the ability to form heat-, solvent- and UV-resistant endospores has only been observed in members of the phylum Firmicutes (low-G+C Gram-positive bacteria, recently renamed Bacillota) (1-8). Occasional reports of endospore-formers from other phyla have not been validated so far $(9,10)$. Sporulation enables bacteria to survive adverse environmental conditions. Thus, when this ability is found in human pathogens that cause severe diseases, such as anthrax, botulism, colitis, infectious diarrhea, gas gangrene, sepsis, tetanus, and food poisoning, it makes them particularly dangerous and difficult to eradicate $(4,5)$. Even spore-formers that used to be considered benign could turn out to be opportunistic pathogens, as exemplified by the recently described involvement of Paenibacillus thiaminolyticus in postinfectious hydrocephalus (11).

Formation of endospores is a complex process that starts with the asymmetric division of a vegetative cell producing a mother cell and a prespore, and proceeds through several stages of spore maturation. In the best-studied model organism Bacillus subtilis, the sporulation process affects expression of more than 500 genes, some of which are essential for sporulation, whereas others appear to be involved in various regulatory circuits (12-20). While some genes are exclusively involved in sporulation and are only expressed at certain stages of the process, spores also contain many housekeeping proteins that function during spore germination and subsequent vegetative growth (21-23). Studies on sporulation mechanisms in clostridia identified a somewhat smaller set of sporulation genes than in bacilli, leading to the conclusion that clostridia encode a streamlined version of the sporulation machinery $(22,24-27)$.

The ability to form spores is widespread in the two major classes of Firmicutes, Bacilli and Clostridia, and also has been observed in the other, more recently described, firmicute lineages (4, 28-30). However, some well-studied taxa within the Bacilli, such as lactobacilli, listeria, staphylococci, and streptococci, do not include any spore-forming members (4). Similarly, no spore-formers have been identified in the clostridial family Halanaerobiaceae (order Halanaerobiales) and in several families of the order Clostridiales (recently renamed Eubacteriales) $(4,31)$. Many other firmicute lineages include both spore-forming and non-sporeforming members (4). 
These observations, coupled with the attempts to identify potential drug targets for extermination of spores, call for identification of the core set of essential sporulation genes that are necessary and, possibly, sufficient for a bacterium to be a spore-former. The most obvious candidate, the sporulation master regulator Spo0A, indeed, appeared to be essential, as no spo0A- organism had been shown to form spores $(4,32)$. However, Spo0A is encoded in the genomes of many non-spore-formers and, therefore, could not be used as a reliable predictor of the sporulation ability $(4,32)$. Three more genes, sspA, dpaA (spoVFA), and $d p a B$ (spoVFB), initially proposed as sporulation signatures (33), presented the same problem, being present in a variety of non-spore-formers (32).

The availability of complete genome sequences of many diverse firmicutes offers an opportunity to identify sporulation genes through comparative genomics. Following the approach pioneered by Stragier (34), several studies took advantage of the constantly expanding genome list to define the conserved core of sporulation genes that are present in all (or at least most) known spore-formers $(2,32,35-37)$. The resulting list included about 60 genes, many of which had been previously shown to be essential for sporulation because mutations in these genes caused sporulation arrest and/or production of immature spores. However, deletion of some other genes from the core set appeared to have only a minor effect on the sporulation efficiency, indicative of a substantial redundancy of the sporulation machinery. Indeed, in $B$. subtilis, many sporulation genes are found in two or more paralogous forms $(37,38)$.

Here, we used the latest update of the Clusters of Orthologous Genes (COG) database (39) to analyze the sporulation gene sets in the current genome collection of diverse firmicutes, including members of the recently defined classes Negativicutes, Tissierellia, Erysipelotrichia, and Limnochordia. The COG database covers a limited number of selected, completely sequenced microbial genomes (typically, a single representative per bacterial genus) and features COG-specific patterns of presence-absence of genes in the respective organisms $(40,41)$. Thus, COG profiles offer an easy way to identify those genomes in which a given gene, e.g. one involved in sporulation, is missing $(39,41,42)$. Analysis of COG profiles of 180 genomes representing 76 spore-forming and 102 asporogenous species allowed us to detect numerous events of lineagespecific gene loss in various components of the sporulation machinery. Of particular interest was the widespread loss of sporulation genes in the families Planococcaceae and Erysipelotrichaceae, which resulted in a greatly streamlined machinery for engulfment, formation 
of the spore coat, and spore germination. These observations suggest that certain members of these families could be excellent model organisms for studying the fundamental mechanisms of sporulation in greater molecular detail.

\section{RESULTS}

Spore-former genome collection. The analyzed set consisted of 180 genomes from 160 genera, representing every named genus of Firmicutes that included at least one completely sequenced genome by April $1^{\text {st }}, 2019$, and several genomes released after that date (39). These organisms belong to six currently recognized classes of Firmicutes: Bacilli, Clostridia, Erysipelotrichia, Limnochordia, Negativicutes, and Tissierellia, and comprise 76 species whose original descriptions mentioned their ability to sporulate, 102 non-spore-formers (based on the descriptions of the respective strains), and two species with an unclear sporulation status (Table $\mathbf{S 1}$ in the Supplemental material). At the time of this study, there were no completely sequenced genomes from any representatives of the classes Culicoidibacteria and Thermolithobacteria; members of these classes have been described as non-spore-forming $(43,44)$.

To ensure reliability of the gene presence-absence patterns, this work relied on high-quality genomes included in the recent release of the COG database (39), most of which had been vetted by the NCBI's RefSeq team and selected as RefSeq reference genomes (45). For two organisms, Caproiciproducens sp. NJN-50 and Thermincola potens JR, the sporulation status had not been described and they had both spore-forming and asporogenous relatives. To ensure proper coverage of the class Erysipelotrichia, its two members featured in the COG database were supplemented with five additional genomes, including two from known spore-formers, [Clostridium] innocuum (the square brackets indicate that this organism has been misnamed, which remains to be rectified (28)) and Erysipelatoclostridium (formerly Clostridium) ramosum $(28,46,47)$.

Sporulation and genome size. As reported previously, spore-formers generally have larger genomes than non-spore-formers (32). In our set, the mean genome size of the former was $\sim 3.9$ $\mathrm{Mb}$, compared to $\sim 2.7 \mathrm{Mb}$ for the latter (Figure S1 in the Supplemental material). Although the genome size distributions overlapped, the previously reported 2.3-Mb boundary for cultivated spore-formers (32) held for the expanded genome set analyzed here. Only two spore-formers in 
the set, both still uncultured, Candidatus Arthromitus SFB-mouse and Candidatus Desulforudis audaxviator MP104C, had genome sizes of less than $2.4 \mathrm{Mb}$ (see Table S1; the recently cultivated spore-forming strain of $\mathrm{Ca}$. Desulforudis audaxviator with a 2.2-Mb genome (48) was not part of the COG set). In agreement with the previous reports $(4,49,50)$, the ability to sporulate often differed even between closely related organisms: families Bacillaceae and Planococcaceae in the class Bacilli, most families in class Clostridia, and the families Tissierellaceae and Erysipelotrichaceae all included both spore-forming and asporogenous members (Table S1). A comparison of the genome sizes of spore-formers and non-spore-formers from different classes is presented in Figure S1B.

Selection of sporulation genes. For the purposes of this work, the 'sporulation genes' were defined as those genes that participate in spore formation but have no known housekeeping roles in vegetative cells. This approach excluded most metabolic enzymes, as well as proteins involved in DNA replication and repair, transcription, translation, motility, secretion, and other processes. However, we included the cell division genes that are (also) involved in asymmetric cell division, as well as Spo0A-regulated genes that function at the onset of sporulation; pre-spore and forespore genes expressed under the control of SigF and/or SigG; mother cell genes that are expressed under the control of SigE and/or SigK; genes involved in the formation of the spore cortex and spore coat; and genes involved in spore germination. The list of the genes belonging to these groups was compiled based on the previous studies $(2,15,16,28,32,36,37)$ and the data from the SubtiWiki database (http://subtiwiki.uni-goettingen.de/) (38). In the COG database, products of these genes formed 237 clusters of orthologs (COGs). We extracted COG phyletic profiles (the patterns of gene presence-absence in the respective genomes) for these sporulation genes and sorted them by their functions and representation among the 76 spore-forming organisms. The list of the most common sporulation genes identified in this manner is presented in Table 1. The patterns of distribution of these genes among all 180 analyzed species are listed in Table $\mathbf{S 2}$ in the Supplemental material and are shown graphically in Figure $\mathbf{S 2}$.

Distribution of spo0A, dpaAB, and sspA genes. In 2004, Weigel and colleagues identified four conserved sporulation genes, namely, spo0A, $d p a A, d p a B$, and $s s p A$, and proposed using these genes as markers of the ability of a bacterium to form endospores (33). We have previously reported the presence of these genes in several clostridial non-spore-formers and argued that the presence of these genes was insufficient - and, in the case of $d p a A B$, unnecessary - for 
sporulation (32). It was instructive to test the distribution of these four genes in the current, much more diverse collection of firmicute genomes.

spo0A. Analysis of the current genome set confirmed that the master cell regulator Spo0A is an essential component of the sporulation machinery. Indeed, the genome of every experimentally characterized spore-forming member of the Firmicutes encodes Spo0A, and its absence is an excellent predictor of the organism's inability to sporulate. Among the 180 species analyzed in this work, the spo0A gene was present in 118, including all 76 spore-formers and 40 non-sporeformers (Table 1, Table S1). These observations confirm that the presence of spo0A is insufficient to conclude that a bacterium is a spore-former although its absence unequivocally indicates that it is not. In agreement with the previous reports $(32,33,51)$, Spo0A-encoding non-spore-formers were found in classes Bacilli, Clostridia, and Erysipelotrichia. Among Bacilli, our set included six Spo0A-encoding non-spore-formers, all in the order Bacillales: Lentibacillus amyloliquefaciens in the family Bacillaceae, Kurthia zopfii and Planococcus antarcticus in Planococcaceae; Macrococcus caseolyticus in Staphylococcaceae, Novibacillus thermophilus in Thermoactinomycetaceae, and Exiguobacterium sp. from Bacillales Family XII Incertae Sedis. No Spo0A-encoding genomes and accordingly no spore-formers were represented among the 23 members of the order Lactobacillales. The current version of GenBank includes several spo0A genes from several clinical isolates of Streptococcus pneumoniae, but it remains unclear whether these isolates are correctly classified.

Spo0A is encoded by 32 of the 42 non-spore-forming members of Clostridia in our set and both members with an unclear sporulation status. Among the ten members of Negativicutes and nine members of Tissierellia, all four Spo0A-encoding organisms were spore-formers. Among the seven representatives of Erysipelotrichia, Spo0A was encoded in two spore-formers, C. innocuum and E. ramosum, and two non-spore-formers, Amedibacterium intestinale and Turicibacter sp. (Table S1). Most Spo0A ${ }^{+}$genomes, 107 out of 118, carried a single spo0A gene. The only exceptions were observed in the order Clostridiales where 10 genomes encompassed two paralogous spo0A genes each, and one, the asporogen Anaerostipes hadrus, had three spo0A paralogs. Irrespective of their ability to form spores, Spo0A-encoding organisms had larger genomes than Spo0A- ones (Figure S1B) and possessed many more sporulation genes than Spo0A $A^{-}$genomes (Figure 1). This trend was most pronounced for widespread sporulation genes (Figure 1A) but also held for those sporulation genes that were conserved mostly in Bacilli (Figure 
1B) and for more narrowly conserved sporulation genes (Figure 1C). The phylogenetic tree of Spo0A proteins (Figure S3 in the Supplemental material) showed only minor deviations from the 16S rRNA-based phylogeny of the Firmicutes, suggesting vertical inheritance of the spo0A genes within this phylum (see below).

$\underline{d p a A B}$. Dipicolinate, a key spore component, is produced by oxidation of dihydrodipicolinate, which is catalyzed by dipicolinate synthase, whose two subunits are encoded by dpaA (spoVFA) and $d p a B$ (spoVFB) genes that are expressed in the mother cell under $\sigma^{\mathrm{K}}$ control. Among the members of the class Bacilli in our genome set, the $d p a A B$ gene pair was found in all sporeformers and was absent in nearly all non-spore-formers. Therefore, within Bacilli, these genes indeed could serve as markers of sporulation. The $d p a A B$ gene pair was also found in Limnochorda pilosa and in both spore-forming members of Erysipelotrichia. In clostridia, however, the picture was more complicated. The $d p a A$ and $d p a B$ genes have been previously shown to be missing in Clostridium perfringens, C. botulinum, and C. tetani, in which dihydrodipicolinate oxidation is catalyzed by the electron transfer flavoprotein, encoded by the etfA-etfB gene pair $(32,52)$. Non-orthologous displacement of $d p a A B$ by etfAB was also observed in several other clostridial spore-formers, including Clostridium acetobutylicum, Alkaliphilus metalliredigens, Paeniclostridium sordellii, and Tepidanaerobacter acetatoxydans, as well as two spore-forming representatives of Negativicutes, Methylomusa anaerophila and Pelosinus fermentans, and in Tissierella sp. JN-28 (aka Sporanaerobacter sp. NJN-17), a member of the class Tissierellia. Finally, Gottschalkia acidurici does not encode either DpaAB or EtfAB, suggesting that in this organism dipicolinate production is catalyzed by yet another, currently unidentified oxidoreductase.

sspA. The distribution of the $\operatorname{ssp} A$ gene, which encodes $\alpha / \beta$-type small acid-soluble sporulation protein (SASP), is also dramatically different between classes Bacilli and Clostridia. Among bacilli, $s s p A$ is found in all spore-formers and is missing in nearly all non-spore-formers (Table S2), making it another good marker of the ability of bacilli to sporulate. Essentially the same picture was observed in the classes Negativicutes, Tissierellia, and Erysipelotrichia. However, in Clostridia, the $\operatorname{ss} A$ gene is represented both in spore-formers and in many non-spore-formers, albeit only in Spo0A-encoding ones. The only spore-former in our set that did not carry the $\operatorname{ssp} A$ gene was $C$. innocuum. The ability of this organism to form spores in the absence of SspA (or any other known SASPs; see below) merits further study. 
Identification of the core sporulation gene set. The COGs that included the $B$. subtilis sporulation genes were sorted by their representation in the 76 spore-former genomes and, specifically, in the genomes of spore-forming bacilli and clostridia. We classified these COGs into four groups (Table 1): i) genes (COGs) that were represented in every spore-former genome, with the possible exception of one or two; ii) genes that were widespread in spore-formers but were missing in three or more genomes, often those from a specific lineage; iii) genes that were widespread in bacilli but poorly represented in other classes, and iv) genes that were only present in a limited number of spore-formers. Representation of these genes (COGs) in all analyzed firmicute genomes was recorded as well (Table S2).

As mentioned above, the analyzed genomes were extracted from the COG collection (39), most of which were selected from the NCBI's RefSeq database (45). Nevertheless, it has been shown that even highly conserved ribosomal proteins are occasionally missed during genome assembly, left untranslated because of sequencing errors, or simply overlooked in the course of genome annotation (53). Therefore, cases of widespread sporulation genes missing in one or more spore-former genomes were individually verified by checking for the potential presence of highly diverged or partial ORFs using DELTA-BLAST search (54) against the NCBI protein database and/or by TBLASTn search (55) against the nucleotide sequence database, each time selecting the respective species or strain as the target database and a representative protein from a closely related organism as a query (56). This analysis identified frameshifts and nonsense mutations in several widely conserved sporulation genes in several genomes (Table S3 in the Supplemental material). However, the occurrence of such suspicious frameshifts was typically limited to one or two per genome, even in Sulfobacillus acidophilus str. TPY (GenBank accession number $\underline{\mathrm{CP} 003179.1}$ ), which had several unannotated ribosomal proteins (53) and was excluded from RefSeq because of the missing rRNA genes. This genome also lacked an unusually high number of widespread sporulation genes (Table S4 in the Supplemental material). Another exception was the genome of Jeotgalibacillus malaysiensis D5 (GenBank: CP009416.1), which also had several frameshifted and missing sporulation genes (Tables S3 and S4), some of which were found in other Jeotgalibacillus spp. (Table S2). The peculiar phylogenetic position of the genus Jeotgalibacillus between Bacillaceae and Planococcaceae $(57,58)$ warranted keeping this genome in the analyzed set but its sporulation genes patterns were considered unreliable. 
Comparison of Table 1 with the previously identified sets of core sporulation genes $(2,32,36$, 37) showed that the list remained largely stable, despite the substantially expanded coverage of Bacilli and Clostridia and inclusion of representatives of three more classes of Firmicutes, Negativicutes, Tissierellia, and Limnochordia (Table S5 in the Supplemental material). Most genes previously assigned to the conserved core were found in all or nearly all of the 76 sporeformers in the current genome set. Further, several genes, such as spolVFB, spoVE, cotS, yfhM, yhaX, yisY, and yyaA, turned out to be more widespread than previously thought (Table S5). The only deviation from this pattern was observed in the class Erysipelotrichia, in which the sporeforming members, $C$. innocuum and E. ramosum, missed more than a dozen genes from the conserved sporulation gene set (see below). This comparison also showed that, despite the overall conservation of the core sporulation genes in all classes of Firmicutes, many of these genes were found to be completely missing (as opposed to being disrupted by frameshifted) in one, two, or more genomes (Table $\mathbf{S 6}$ in the Supplemental material). Although some of these gaps in phyletic patterns could be caused by errors in genome sequencing and/or assembly, the consistency of such patterns in certain phylogenetic groups appeared to reflect an actual evolutionary trend. In the following sections, we describe the streamlining of the sporulation gene complement in spore-formers from families Planococcaceae and Erysipelotrichaceae, followed by the discussion of the gene loss within specific sporulation systems.

Sporulation genes in Planococcaceae. The analyzed genome set included six spore-forming members of the family Planococcaceae (also known as Caryophanaceae), order Bacillales: Paenisporosarcina sp. K2R23-3, Rummeliibacillus stabekisii, Solibacillus silvestris, Sporosarcina psychrophila, and Ureibacillus thermosphaericus. In addition, we included Jeotgalibacillus malaysiensis, which is currently assigned to the Planococcaceae, and Lysinibacillus sphaericus, which is usually assigned to Bacillaceae but in phylogenetic analyses robustly falls within the Planococcaceae (58). These seven organisms showed a consistent pattern of sporulation gene loss, with most of them lacking four of the nine components of the SpollQ-SpollIA complex (spollIAA, spollIAB, spollIAD, and spollIAF genes), as well as such conserved sporulation genes as spmA, spmB, spollM, gerM, gerW (ytfJ), yqfC, and ytxC, which are found in most spore-formers (Tables 1, S2, S4). Furthermore, these seven organisms also lacked some genes that are generally conserved among the spore-forming members of the Bacillaceae: bofC, $c s f B$, spolIIL, spoIVFA, spoVAA, spoVAB, spoVAEA, spoVID, yabG, yhbB, yrrD, ysxE, and ytxC (Table S2). 
They also lack the genes that encode such SASPs as SspK, SspL, SspN, SspO, SspP, and CsgA; spore coat proteins CotM, Cotl, CotO, CotP, and CotW, and spore maturation proteins SpsJ and CgeB, which are found in most spore-forming bacilli (Table S2). The most dramatic deviations from the common phyletic pattern were detected in J.malaysiensis, in accordance with its uncertain phylogenetic position (58). The consistent absence of all these genes suggests that members of the Planococcaceae possess substantially streamlined sporulation machinery. The analyzed genome set also included two asporogenous representatives of Planococcaceae, $K$. zopfii and $P$. antarcticus, which have spoOA but lack almost all other sporulation genes (Table S2).

Sporulation genes in Erysipelotrichaceae. The evolution of the Erysipelotrichia lineage likely involved massive loss of genes, including many related to sporulation (59). Indeed, the genomes of $C$. innocuum and E. ramosum, despite their relatively large size (4.7 and $3.25 \mathrm{Mb}$, respectively), show patterns of extensive loss of sporulation genes. Consistent with the previous observations $(59,60)$, they both lack seven of the nine genes of the SpolIQ-SpollIA complex, retaining only SpolIQ and SpollIAH components. Further, they both lack such widely conserved sporulation genes as spollID, bofA, cotQ, safA, yabQ, ypeB, yyaC, yyaD, sleL (yyaH), and gerW (ytfJ) (Table S2, Table S4). Despite the evolutionary proximity of Erysipelotrichia and Bacilli $(28,59)$, many widely conserved bacillar sporulation genes are missing as well, including bofC, csfB, spollIL, spolVFA, spoVAA, spoVAB, spoVAEA, spoVID, yabG, yhbB, yrrD, ysxE, and ytxC. Some sporulation genes appear to have been lost in only one of these genomes. In particular, $C$. innocuum lost spolIR and spoVS, whereas E. ramosum retained these genes but lost spoVG and spoVT (Table S4). Another system with an unusual phyletic pattern is the spore germination receptor GerABC. While non-spore-forming members of Erysipelotrichia either encode all three subunits of GerABC (Turicibacter sp. H121) or none at all (three other genomes), the sporeformers $C$. innocuum and E. ramosum encode the membrane subunit GerA (GerAA) but not the membrane transporter GerB (GerAB) or the lipoprotein GerC (GerAC) subunits. Although the GerABC receptors are not strictly required for germination (they are missing in Clostridioides difficile $(3,61)$ and could be dispensable in $C$. botulinum (62)), they are nearly ubiquitous among spore-formers (Table S2). The presence of GerAA but not GerAB or GerAC subunits is so far unique among known spore-formers. 
Sporulation onset and asymmetric division. Along with spo0A, genes involved in sporulation onset and asymmetric division are highly conserved in spore-formers of the class Bacilli, and many, although not all, of these genes, are also conserved in spore-forming members of Clostridia and four other classes of Firmicutes (Table 1, Table S2). As discussed previously, many clostridia lack the Spo0B-Spo0F-Spo0A phosphorelay system, and their Spo0As are phosphorylated by orphan histidine kinases that are not orthologous to any of the five sporulation histidine kinases (KinA - KinE) of B. subtilis. However, many of these orphan kinases contain at least one PAS sensor domain $(25,51,63)$. Nevertheless, the spoOB-spoOF gene pair is present in several clostridia, primarily members of the families Heliobacteriaceae, Peptococcaceae, and Thermoanaerobacteraceae (51), as well as in Negativicutes and Tissierellia (Table S2).

The genes involved in asymmetric cell division, such as $\operatorname{divIB}, \operatorname{divIC}, \operatorname{divIVA}, \mathrm{fts} E, \mathrm{fts} H, \mathrm{fts} X$, $f t s Y, f t s Z, \min C, \min D$, and $\operatorname{minJ}$, are widespread in bacteria, including most firmicutes and nearly all spore-formers (Table S2). As expected, genes encoding the four sporulation-specific sigma subunits, sigF, sigG, sigE and sigK, are found in every spore-former, but the Spo0A-regulated genes spollAA, spollAB, spollE, spollGA, spo0JA (parA), and spo0JB (parB) are also highly conserved in spore-formers of every firmicute lineage (Table 1). However, such Spo0Adependent genes of $B$. subtilis as $y k u J, y k u K$, and $y n e F$, all three with unknown functions, are not conserved even within Bacillales (Table S2).

Formation and activation of $\sigma^{\mathrm{K}}$. While $\sigma^{\mathrm{K}}$ is universally encoded in the genomes of all sporeforming firmicutes, its activation is tightly controlled, preventing premature expression of latestage sporulation genes in the mother cell (64-66). In B. subtilis strain 168, the $\sigma^{\mathrm{K}}$-encoding gene is interrupted by a 48-kb prophage-like skin (sigk-intervening) element, which results in two separate coding regions denoted spoIVCB and spollIC, neither of which is expressed. These fragments of sigK are joined as a result of SpolVCA-dependent excision of skin to form the complete, intact sigK gene that encodes pro- $\sigma^{\mathrm{K}}(67,68)$. Pro- $\sigma^{\mathrm{K}}$ is then activated in the mother cell through cleavage by the intramembrane metalloprotease SpolVFB $(64,66)$. In C. difficile 630 , the skin element is only $14.6 \mathrm{~kb}$ long, and its excision yields a sigK gene encoding a mature $\sigma^{\mathrm{K}}$, which does not require proteolytic activation $(69,70)$. SigK-interrupting skin elements have also been identified in $C$. tetani and $C$. perfringens $(71,72)$. We confirmed the presence of split sigK genes in B. subtilis, C. difficile, and C. tetani, and identified split sigK genes in 12 additional organisms from Bacilli, Clostridia, Negativicutes, and Tissierellia (Figure 2). These skin elements 
range in size from 2.6 to $48 \mathrm{~kb}$ and encode from 2 to 67 proteins (see Table $\mathbf{S 7}$ in the Supplemental materials).

The orientation of skin elements with respect to the sigK component genes is variable, but the spolVCA gene is always located at the edge of the element, usually immediately upstream of the spollIC gene, which encodes the C-terminal fragment of $\sigma^{\mathrm{K}}$ (Fig. 2). The SpoIVCA proteins from different skin elements (see Figure S4A in the Supplemental materials for a sequence alignment) are members of a single family of site-specific DNA recombinases (COG1961) that is represented by multiple copies in most firmicute genomes, including up to 26 genes in (non-spore-forming) Flavonifractor plautii and Oscillibacter valericigenes. In the phylogenetic tree of SpolVCA-like recombinases/integrases (Figure S4B), SpolVCAs from skin elements are interspersed with those from tailed phages (Caudovirales), suggesting (pro)phage origin of the skin elements. Remarkably, aside from spollIC, spoIVCA and spoIVCB, no genes are shared by all skin elements (Figure S4C). The presence of skin elements that split sigK genes in two non-adjacent fragments often causes confusion in genome annotation. Thus, in the current genomic entries for $B$. subtilis strain 168 (Genbank accessions AL009126.3, CP051860.2, CP053102.1, and CP052842.1), sigK is erroneously marked as a pseudogene, whereas in genomic entries for C. tetani E88 (GenBank: AE015927.1) and Pelosinus fermentans (GenBank: CP010978.1), the C-terminal spollIC-like fragment is left untranslated (Tables S5, S7). Remarkably, sigK is not unique as the skin insertion site: skin-like elements were also found interrupting the cotJC gene of Laceyella sacchari and spollID gene of Ruminiclostridium cellulolyticum (Table S3).

In $B$. subtilis, activation of pro- $\sigma^{\mathrm{K}}$ by SpolVFB is regulated by BofA, BofC, and also SpolVFA, which is itself regulated by proteolysis by the forespore-expressed protease SpolVB (73). $\sigma^{\mathrm{K}}$ is encoded in every spore-former genome as is, with a single exception, SpolVFB, which is a member of a widespread metalloprotease family (COG1994). However, SpolVFB-interacting BofA, BofC, and SpolVFA are far less widespread. BofA is missing in 10 of the 76 spore-formers, including six clostridia, C. innocuum, E. ramosum, and Limnochorda pilosa. The distribution of BofC and SpolVFA is generally limited to the members of the class Bacilli. The full-length $B$. subtilis-like BofC, which consists of two distinct domains (74), is found almost exclusively in the families Bacillaceae and Paenibacillaceae; it is missing in some members of the former and, conspicuously, in members of the Planococcaceae (Table S2). The C-terminal domain of BofC, PF08955, is more widespread but is still missing in most spore-forming clostridia. SpolVFA is 
missing in the Planococcaceae, in nearly all clostridia, and, again, in C. innocuum, E. ramosum, and L. pilosa. Remarkably, SpoIVFA-cleaving serine protease SpolVB is nearly universal among spore-formers, being represented in numerous genomes that do not encode SpoIVFA. The details of SpolVFB-BofA interaction in the absence of SpolVFA, and the functions of SpolVB homologs in these organisms remain to be elucidated.

Conservation of the engulfment complex. In $B$. subtilis, engulfment is triggered by SpollB and involves two key protein complexes, SpolID-SpolIM-SpolIP (the DMP complex) and SpollIASpolIQ, which are targeted to the growing septum by either SpollB or the SpoIVFA-SpolVFB pair (75-77), with additional involvement of GerM (78). Both protein complexes are found in C. difficile (79) and are conserved in almost all spore-formers (Table 1), whereas the targeting proteins are less widespread: as noted previously (32), both spolIB and spoIVFA are missing in clostridia (Table S2).

Among the three genes encoding the peptidoglycan-degrading DMP complex, spollD was missing in a single genome in our 76 spore-former genome set, and spollP was missing in two (Table S6); these three cases could be due to the sequencing problems. In contrast, spollM, although widespread as well, was missing in nine genomes (Table S2). This pattern is consistent with the reports that SpolIM is not essential for engulfment in C. difficile $(80,81)$, whereas SpolID and SpollP appear to be indispensable.

The transmembrane SpollIA-SpollQ complex consists of nine proteins, eight of which are encoded in the mother cell by the genes of the spoIIIAA-AB-AC-AD-AE-AF-AG-AH operon that is under the SigE control. The ninth protein, SpolIQ, is produced in the forespore under SigF control and contains the M23-type metallopeptidase LytM domain $(82,83)$. The core of the SpollIASpolIQ complex consists of SpollIAH and SpollQ proteins. Both these proteins form dodecameric (or even larger) rings that interact to form a $60 \AA$ (or even a $140 \AA$ ) transmembrane channel, which connects the mother cell and the forespore and enables trafficking of small molecules between the two compartments $(84,85)$. The structure of this complex from B. subtilis (Figure S5A) revealed a key role of the LytM domain of SpolIQ in its interaction with SpollIAH. However, despite the presence of the common LytM domain, SpolIQ proteins from bacilli and clostridia are substantially distinct: the M23 peptidase is inactivated in the former but appears to be intact in the latter (86). Accordingly, these proteins belong to two different COGs, COG5820 and COG5821, 
in the COG database (39). Both varieties of SpollQ are often encoded in similar spol/D-spol/QspollID operons although the genomic neighborhoods of spollQ are highly variable even within the Bacillaceae (Figure S6). Although we previously reported not being able to find orthologs of clostridial spollQ (CD0125 in C. difficile) in the genomes of several members of the family Peptococcaceae (32), in this work, using synteny and DELTA-BLAST (54) searches, we identified these in the genomes of all clostridial spore-formers but very few non-spore-formers (Table S2). Spore-forming members of Erysipelotrichia, C. innocuum and E. ramosum, encode the bacillar form of SpollQ, whereas spore-forming members of Negativicutes, Tissierellia, and Limnochordia encode the clostridial form. Thus, all 76 spore-formers in our set encode both SpollIAH and one of the two forms of SpollQ, indicating strict conservation of the SpollQ-SpollIAH pore among spore-forming bacteria.

As noted above, two groups of Firmicutes, Erysipelotrichaceae and Planococcaceae, have apparently lost multiple components of the SpollQ-SpollIA complex. The Erysipelotrichia members $C$. innocuum and $E$. ramosum retain only the spolIIAH and spollQ genes. In addition, they possess SpolVFB and GerM, but lack SpollB and SpolVFA (Table S2, Figure S5B, C), see also reference (60). Six members of the family Planococcaceae show stronger conservation of the SpolIQ-SpollIA complex: in addition to SpollIAH and SpolIQ, they retain SpolIIAC, SpollIAE, and SpollIAG components (Fig. S3). However, all these organisms lack SpollB, SpolVFA, SpolVFB, and GerM proteins that participate in the assembly of the SpolIQ-SpollIA complex in B. subtilis. Members of Planococcaceae, such as Lysinibacillus sphaericus, could be useful model organisms for studying the core mechanisms of engulfment in the Firmicutes.

Small acid-soluble sporulation proteins (SASPs). SASPs are short proteins that bind doublestranded DNA and protect spores from heat, UV radiation, and other adverse conditions (87-91). In $B$. subtilis, the majority of the SASPs come from two families, $\alpha / \beta$ (COG5852, which unifies SspA, SspB, SspC, and SspD families, and COG5854, SspF) and gamma (COG5853), whereas several minor SASPs $(87,90,92)$ comprise COGs from COG5855 through COG5864. As observed previously, the $\alpha / \beta$-type SASPs are nearly universal in spore-forming firmicutes, whereas the distribution of other SASP families varies $(32,93)$. This observation is supported by the data from our new set. Indeed, COG5852 is represented in 75 of the 76 spore-formers and in 31 of the 40 SpoOA-encoding non-spore-formers (Table S2). The only spore-former missing SspA was C. innocuum; the other spore-forming member of Erysipelotrichaceae, E. ramosum, has two 
sspA-like paralogous genes and a single sspl gene for a minor SASP. Homologs of sspA are also found in other members of Erysipelotrichaceae, such as [Clostridium] spiroforme and [Clostridium] cocleatum (Figure S7). However, C. innocuum lacks known ssp genes, so the nature of its SASPs, if any, remains enigmatic. Most of the minor SASPs described in B. subtilis are also encoded in at least some members of Bacillales (93). In contrast, members of other classes of Firmicutes only encode $\alpha / \beta$-type SASPs of the SspF and Ssp4 families (94, 95); some clostridia also encode TIp-like SASPs. Thus, the diversity of SASPs in B. subtilis does not appear to be shared by other groups of Firmicutes, which is consistent with the reports of non-essentiality of minor SASPs $(88,95)$.

Spore cortex. Genes involved in the biosynthesis of the spore cortex, the peptidoglycan layer that surrounds the forespore membrane, have been previously found to be conserved throughout Bacilli and Clostridia (32). Examination of the current expanded genome set showed that this conclusion still holds for spoVB, spoVD (ftsl), spoVE (ftsW), spoVV (ylbJ), yabP, and yqfD genes (Table 1). One more gene, yabQ, which is essential in $B$. subtilis $(96,97)$, was only missing in the genomes of $C$. innocuum and $E$. ramosum, whereas the yqfC gene was missing in the genomes of six Planococcaceae members and E. ramosum (Table S6).

Spore coat. As noted previously $(32,98,99)$, bacilli and clostridia have dramatically different spore coats: many of the $\sim 70$ proteins that form the coat of $B$. subtilis (7) are only conserved within Bacillaceae, or not even in all members of this family $(32,100)$. Among the ten morphogenetic spore coat proteins of $B$. subtilis (SpolVA, SpoVM, SpoVID, SafA, CotE, CotH, CotO, CotX, CotY, and CotZ), only SpolVA is universally conserved in all spore-forming firmicutes (Table S2). In bacilli, SpoIVA interacts with SpoVID $(101,102)$, whereas in C. difficile and some other clostridia, its interaction partner is SipL (CD3567), which shares with SpoVID a common Cterminal LysM domain $(103,104)$. Of the other SpolVA- and/or SpoVID-interacting proteins, SpoVM is found in some clostridia but is not essential for sporulation (105), CotE is found only in a few clostridia, and the full-length SafA (as opposed to its LytM domain) is not represented outside of bacilli. CotH, CotO, CotX, CotY, and CotZ are missing even in some members of Bacillaceae and, except for $\mathrm{CotH}$, none of these genes is found in clostridia or members of other classes of Firmicutes (Table S2). 
Among the proteins of $B$. subtilis inner coat, the most widespread are proteins of the CotJC/YjqC family, which belong to the COG3546 "Mn-containing catalase". Three members of this family (CotC, CotD, and CotE) were found in the spore coat of C. difficile, and the latter two have been shown to retain enzymatic activity $(98,106)$. Two more spore coat proteins of C. difficile, peroxiredoxin CotE and superoxide dismutase SodA, could be involved in protection from oxidative stress (98). Other widely conserved proteins of the $B$. subtilis basement layer and inner coat are also (not necessarily active) enzymes: acetyltransferase CgeE, peptidoglycan hydrolases CwIJ and SleL (YaaH), phospholipase LipC, HAD family phosphatase YhaX, ATPgrasp family enzymes $\mathrm{YheC}$ and $\mathrm{YheD}$, MenH-related esterase YisY, and glycosyltransferases CotSA and YdhD (Table S2). A similar pattern is apparent in the outer coat, which contains alanine racemase $Y n c D$, nucleotidyl transferase $Y t d A$, predicted $F A D$-dependent dehydrogenase CotQ, and LysM domain-containing YkzQ. Both inner and outer coats of $B$. subtilis contain members of the small heat shock protein (HSP20) family, CotP and CotM, respectively. These examples demonstrate the widespread recruitment (exaptation) of common housekeeping enzymes as structural components of the spore coat. Their broad representation among bacillar sporeformers, as opposed to the scarcity in non-spore formers (Table S2), suggests that such recruitment is a common mechanism of spore coat assembly in Bacilli. There is still insufficient data on spore coat proteins in Clostridia and none on those in other classes of Firmicutes, but studies in C. difficile $(98,106)$ suggest that such recruitment occurs in clostridia as well.

Germination proteins. As mentioned above, the common germination receptors consisting of GerA, GerB, and GerC components are encoded in all classes of Firmicutes (Table S2). In our set of 76 spore-formers, the only exceptions were two members of the family Peptostreptococcaceae, C. difficile and Paeniclostridium sordellii, which lacked all three components of the receptor, and two members of Erysipelotrichaceae, C. innocuum and E. ramosum, which lacked GerB and GerC. In C. difficile, the bile acid germinant receptor has been identified as the pseudoprotease CspC $(99,107,108)$, a member of the vast family of subtilisin-like serine proteases (COG1404). The presence of multiple members of this protein family in most sporeforming firmicutes suggests that the $\mathrm{CspC}$-dependent germination mechanism is not limited to $C$. difficile and could potentially function in other organisms, as an addition to the better-studied GerABC-mediated signaling. 
The germination process involves degradation of the spore protective layers, the cortex, and the inner and outer coats, as well as hydrolysis of DNA-shielding SASPs. In B. subtilis, hydrolysis of the spore cortex is catalyzed by peptidoglycan hydrolases CwlJ and SleB, members of the same COG3773, which is widespread in spore-forming bacilli and clostridia and is also found in spore-forming members of Tissierellia and Limonochordia, albeit not in the Negativicutes or C. innocuum (Table S2). The regulators of the activity of these enzymes, however, are less widespread. YpeB, which regulates SleB activity in bacilli and many clostridia (109-111), is missing in C. difficile and several other clostridia, as well as in all members of Negativicutes and Erysipelotrichia in the current set (Table S2). In C. difficile, the principal spore cortex-hydrolyzing enzyme is SleC (CD630_05510), a multidomain protein that consists of a structurally, but not functionally, characterized DUF3869 domain [Pfam (112) domain PF12985] (113), a SpolID-like amidase domain, and a C-terminal peptidoglycan-binding (PF01471) domain. SleC homologs are found in many clostridia, as well as $C$. innocuum and $E$. ramosum.

Degradation of SASPs is catalyzed by the germination protease, Gpr, an aspartate protease $(95,114)$ that is universally conserved in spore-forming firmicutes (Table 1) but is absent in nonspore-forming members of Bacilli, Negativicutes, Tissierellia, and Erysipelotrichia. Among the clostridial non-spore-formers, the distribution of Gpr is generally similar to that of Spo0A: Spo0Aencoding spore-formers usually have the gpr gene, whereas those genomes that lack spoOA also lack gpr (Table S2).

Among other genes involved in spore gemination in $B$. subtilis, only a few, such as spoVAD, gdh, gerF (lgt), ykvU and ypeB, are widespread, whereas others, such as $\operatorname{csg} A$, gerD, gerE, gerPA/gerPF, gerPB, gerPC, gerPD, gerPE, and spoVAEA, are found mostly (or exclusively) in spore-forming bacilli (Table S2).

Uncharacterized sporulation proteins. Despite numerous studies on sporulation in $B$. subtilis and other model organisms, functions of several widespread sporulation genes have been clarified only recently whereas functions of several others remain unknown (Table 1). Altogether, the SubtiWiki database (38) lists $>250 y$-genes that appear to be specifically expressed during sporulation $(15,16,20,115,116)$, but have not been characterized experimentally. Assignment of such genes to the COGs allowed making at least tentative functional predictions for several $y$ genes (Table S8 in the Supplemental material). Examples include YdgB and YteA proteins, both 
annotated as "uncharacterized" in UniProt, which belong to the COG1734 "RNA polymerasebinding transcription factor DksA“, and the uncharacterized proteins YqcK and YqjC that fall into COG0346 "Catechol 2,3-dioxygenase-related enzyme, vicinal oxygen chelate (VOC) family". Experimental characterization of these genes and other widespread genes of unknown function could provide further insight into the details of sporulation mechanisms in diverse firmicutes.

Evolution of sporulation. We employed the updated list of core sporulation genes (Table 1) to investigate the evolution of sporulation and compare it with the overall phylogeny of Firmicutes. We started by constructing a phylogenetic tree for Spo0A, the master regulator of sporulation that is encoded in each of the 76 spore-formers in our genome collection, as well as 40 non-sporeformers and 2 organisms with uncertain sporulation status (Table S1). Several attempts to construct maximum-likelihood trees from an alignment of full-length SpoOA sequences using MEGA X (117), with several different sets of parameters, resulted in trees where proteins from the members of the same bacterial family typically formed separate clades. However, these trees could not be considered fully reliable because many branches were weakly supported (data not shown). By contrast, removing the variable-length linkers connecting the $\mathrm{N}$-terminal receiver (REC) domain of SpoOA with its DNA-binding domain and using IQ-TREE 2 (118), which automatically selected the best-fit model (119), resulted in a well-supported tree (Figure S3). Again, Spo0As from the members of the same bacterial family typically formed separate clades. Among the Bacilli, monophyly was observed for the families Alicyclobacillaceae and Planococcaceae, Spo0As from members of Bacillaceae and Paenibacillaceae each formed two separate clusters (Figure S3). In Clostridia, Spo0As from most members of the families Clostridiaceae, Lachnoospiraceae (formerly known as Ruminococcaceae or Hungateiclostridiaceae), and Peptostreptococcaceae (C. difficile and Paeniclostridium sordellii), as well as from the order Halanaerobiales, appeared to be monophyletic. Spo0As from Peptococcaceae and Oscillospiraceae each formed two separate clusters, while Spo0As from Thermoanaerobacteraceae were found through the entire clostridial branch. At the class level, Spo0As from spore-forming members of the Erysipelotrichia formed a strongly supported clade that mapped within the Bacilli, while members of the Negativicutes (family Sporomusaceae) and Tissierellia mapped within the clostridial clade (Figure S3).

Notably, the monophyly of Spo0As from the members of the same family did not depend on whether they came from spore-formers or non-spore-formers. Thus, Spo0As from all members of 
Planococcaceae formed a single clade, with Spo0As from non-spore-formers Kurthia zopfii and Planococcus antarcticus grouping together with SpoOAs from planococcal spore-formers (Figure S3). Likewise, Spo0A from the spore-former Lachnoclostridium phytofermentans belongs to a clade with non-spore-forming members of the family Lachnospiraceae, whereas Spo0A from the non-spore-former Dehalobacter sp. CF mapped together with spore-forming members of the family Peptococcaceae (Figure S3).

To gain further insight into the evolution of sporulation, and Firmicutes in general, we constructed a ribosomal proteins-based phylogenetic tree for all 180 analyzed organisms as well as sporulation proteins-based and ribosomal proteins-based trees for the 76 spore-formers. The maximum-likelihood phylogenetic tree of 180 firmicutes (Figure S8) was built using IQ-TREE 2 (118) from a concatenated alignment of 54 ribosomal proteins (6951 total positions, 6366 unique) essentially as described previously $(28,120)$. This tree had excellent bootstrap support and largely agreed with the currently accepted 16S rRNA gene-based phylogeny of the Firmicutes. Members of most previously defined Firmicute families clustered together, typically as clades. The notable deviations from the current taxonomy (56) included members of the class Erysipelotrichia (family Erysipelotrichaceae), mapped within Bacilli as a sister group to Listeriaceae and Lactobacillales, and members of three other classes mapping within Clostridia as follows: a) all three families of class Tissierellia as a sister group to Peptostreptococcaceae; b) members of the class Negativicutes as a sister group to families Peptococcaceae and Syntrophomonadaceae, and c) Limnochorda pilosa, the sole member of the class Limnochordia, as a sister group to Sulfobacillus acidophilus and Thermaerobacter marianensis (Eubacteriales Family XVII. Incertae Sedis). This tree also suggested placement of some organisms with previously obscure taxonomy: Intestinimonas butyriciproducens mapped into Oscillospiraceae, whereas Ndongobacter massiliensis and Ezakiella massiliensis fell within the radiation of the family Peptoniphilaceae (class Tissierellia).

For a bird's eye view of the phylogeny of sporulation, we constructed a maximum-likelihood tree based on a concatenated alignment of 41 core sporulation proteins (as listed in Table 1) from all 76 spore-formers in the current set (Figure $3 \mathbf{A}$ ) and compared it with the ribosomal proteinsbased tree for the same 76 species (Figure 3B). This comparison showed a strong, clear trend: with a few exceptions, sporulation protein sets from members of the same family formed wellsupported clades. As an example, members of the Planococcaceae in both trees formed a sister 
group to the Bacillaceae, the only differences being the branching order of Sporolactobacillus terrae, a member of the family Sporolactobacillaceae, and Alkalihalobacillus (formerly Bacillus) halodurans, and two members of the Erysipelotrichaceae, C. innocuum and E. ramosum. The clostridial portions of both trees had closely similar topologies with essentially the same branching order for most groups. Members of the recently recognized classes Negativicutes (family Sporomusaceae), Tissierellia, and Limnochordia mapped within the clostridial lineage, but, again, in the same positions on both trees (Figure 3). The congruence of both trees indicated that the sporulation genes largely evolved by vertical inheritance, without substantial horizontal gene transfer.

The evolutionary history of gains and losses of these sporulation genes (the same 41 except for spolIQ) was reconstructed using GLOOME, by mapping the patterns of gene presence and absence in each lineage onto the phylogeny of 180 representative Firmicutes, to infer the posterior probabilities of gene presence in the ancestral tree nodes (121). This reconstruction placed all 40 core sporulation genes at the root of the Firmicute phylogenetic tree and suggested only a few gene gains (jag in three Lactobacillus spp, the dipicolinate transporter-encoding spoVV/yjiH/ylbJ genes in several non-spore-formers, ycaP in Veillonella and Megasphaera). An interesting example of a likely gain was the spo0A gene in Macrococcus caseolyticus, one of the few instances of this gene in non-spore-forming bacilli and the only such gene in the Staphylococcaceae members in our set (in GenBank, spoOA is also found in the members of the newly recognized genus Mammaliicoccus). The maximum-likelihood approach implemented in GLOOME considered gain of SpoOA by $M$. caseolyticus more likely than the loss of this gene in five other staphylococcal genomes although the highly degraded sequence of this gene and its unusual placement in the phylogenetic tree of SpoOA (Figure S3) suggested the latter alternative as more realistic.

With all 40 core genes mapped to the root of the Firmicutes, GLOOME predicted extensive loss of sporulation genes in non-spore-forming lineages, such as lactobacilli, staphylococci, and listeria. A major loss of sporulation genes was also inferred for the asporogenous members of the order Bacillales, such as Salimicrobium jeotgali, Gemella haemolysans, and Exiguobacterium sp. (see Table S1). A similar picture was observed in the classes Negativicutes and Tissierellia. Many sporulation genes are conserved in the spore-formers Methylomusa and Pelosinus in the former class, and Gottschalkia and Tissierella in the latter, whereas asporogenous representatives of 
these classes retained very few sporulation genes (Table S1). Accordingly, GLOOME interpreted the absence of these genes in the non-spore-formers as lineage-specific gene losses. The two lineages mentioned above, Planococcaceae and Erysipelotrichaceae, showed loss of some sporulation genes in their spore-forming representatives and SpoOA-encoding non-spore formers, and a far more extensive loss in the Spo0A- bacteria and non-spore-formers.

Among Clostridia, massive, family-wide gene loss was only seen in Eubacteriaceae, as most other families included both spore-forming and asporogenic genera. Here, dramatic differences in sporulation gene content were often observed within the same family. Thus, in the family Peptostreptococcaceae, the analyzed genome set included five members, two spore-formers, C.difficile and Paeniclostridium sordellii, and three non-spore-formers, Acetoanaerobium sticklandii, Filifactor alocis, and Peptoclostridium acidaminophilum (49). Accordingly, the first two encompassed near-complete sets of core sporulation genes (with the exception of those coding for the germination receptor GerABC and several others, see above), whereas the three nonspore formers showed the loss of at least 29 of the 40 analyzed genes (see Table S2). Further, many clostridial non-spore-formers still carried spoOA and retained a fair number of sporulation genes (Figure 1, Table S1). As an example, all six representatives of the order Halanaerobiales in the current set were non-spore-formers, but they all carried $s p o 0 A$, and a major loss of sporulation genes was observed only in Halanaerobium hydrogeniformans (Table S1). This case, as well as the examples of lineage-specific gene loss in Butyrivibrio proteoclasticus (family Lachnospiraceae) or Fastidiosipila sanguinis and Mageeibacillus indolicus (family Oscillospiraceae) (Tables S1 and S2), show that dramatic loss of sporulation genes can occur on relatively short evolutionary distances.

\section{DISCUSSION}

Sporulation is a complex process that involves dozens of genes that function in a tightly regulated fashion to ensure the survival of the bacterial cell under adverse conditions. Spores are resistant to a variety of damaging factors, including extreme conditions, and can persist for thousands - and potentially millions - of years (122). A recent study suggests that spores of $B$. subtilis could even survive on a simulated Martian surface (91). A better understanding of the sporulation mechanisms could help in finding new approaches to eradicate spore-forming human and animal pathogens. Furthermore, recent studies of the human microbiome have revealed a 
variety of spore-formers that remain to be characterized in detail (123), suggesting that the impact of Firmicute spore-formers on human health could be even greater than currently appreciated.

An essential step towards understanding the sporulation process is delineation of the set of genes that are necessary and, possibly, sufficient for sporulation across the entire diversity of bacterial spore-formers. Given that the functions of many genes involved in sporulation remain unknown, those genes that belong to the core set would become priority targets for structural and functional characterization. A major stumbling block for the identification of the core set of sporulation genes is the uncertainty of the sporulation status of many bacteria with sequenced genomes. While many genomes come from well-characterized type species, many others do not, and it is usually not known whether the sequenced strain exactly corresponds to the one that was described previously, in some cases, many years ago. A case in point is Acetohalobium arabaticum, which was initially described as a spore-former (124), but apparently lost the ability to sporulate during the laboratory passages $(31,125)$; we listed it as a non-spore-former (Table S1). Conversely, Turicibacter sanguinis, a Spo0A-encoding member of class Erysipelotrichia, was originally described as asporogenous (126) but subsequently shown to form typical subterminal spores (see Extended Data Fig. 4 in ref. (123)). The genome of Turicibacter sp. H121 included in this work has almost the same set of sporulation genes as $C$. innocuum and $E$. ramosum but lacks, among others, divIVA, dpaB, spollGA, spoVK, and spoVN (Table S2), making this strain unlikely to sporulate. The spore-forming representatives of Erysipelotrichia analyzed in this work, C. innocuum and E. ramosum, were occasionally described as forming very low numbers of spores $(127,128)$. Nevertheless, there was no doubt that these organisms are spore-formers, which made defining their set of sporulation genes a worthwhile exercise.

Previous studies by several independent groups (2, 32, 34-37) identified essentially the same set of $\sim 60$ sporulation genes of $B$. subtilis that were shared by spore-forming members of Bacilli and Clostridia. However, in the genomes of some spore-formers, certain genes from this conserved core were found to be missing $(32,36,37)$. The goal of this work was to reassess the distribution of the key sporulation genes using the widest possible selection of complete genomes from all branches of the Firmicutes. To this end, we employed the latest version of the COG database (39) and supplemented it with five recently sequenced genomes from various members of the Erysipelotrichia. This resulted in a set of 180 species from 160 genera, representing 45 different families from six classes of the Firmicutes. At this scale, most genera were represented 
only by a single genome of a single species, which provided a different perspective from most previous studies $(2,32,34-37)$. Therefore, this work complements, rather than supersedes, those studies.

As discussed elsewhere, the COG database displays the patterns of presence-absence of orthologous genes (COG members) across all covered genomes and therefore allows straightforward identification of those genes that are missing in a given genome $(39,42)$. However, there is an important caveat: a single COG often includes several paralogous genes, which can give the impression that each of these paralogs has a wider phylogenetic distribution than it actually does. For example, $c w / J$ and sleB, members of COG3773 "Cell wall hydrolase CwlJ", are both shown as universal (Table 1), although some genomes, such as $C$. perfringens or $C$. difficile, may encode only a single member of that family. Likewise, dacB and dacF are paralogous genes that encode closely related D-alanyl-D-alanine carboxypeptidases (penicillinbinding proteins $5^{*}$ and 1 , respectively, members of COG1686) that are involved in peptidoglycan metabolism. However, the former is expressed in the mother cell under SigE control, whereas the latter is expressed in the prespore under the control of SigF and/or SigG. Members of this COG, which also contains sporulation-independent paralog, dacA, are found in most firmicutes, both spore-forming and not.

Further, certain gaps in phyletic patterns can arise from errors in genome sequencing and annotation. In some cases, genes that were frameshifted in the examined genomes (Table S3) were found in full-size versions in the genomes of other strains or closely related species. Although we made an effort to address the potential annotation problems, fixing possible sequencing errors was not part of this project. As a result, we have recorded the instances of rarely missing genes in Table S6, but were mostly interested in cases where a certain gene was missing in more than one or two genomes, particularly when these genomes came from the same bacterial lineage.

In our previous work, we noted the absence of many key sporulation genes in the relatively large (4.8 Mb) genome of Lysinibacillus sphaericus C3-41 (129), but blamed it on the poor quality of the genome sequence, calling it "the worst offender" among all bacilli and excluding it from most analyses (32). Now, many of those genes were also found to be missing in the genome of the type strain of this species, L. sphaericus DSM $28^{\top}$. Further, such genes as spollB, spolIM, 
spollIAA, spollIAB, spollIAD, spollIAF, spoVAA, spoVAB, spoVID, bofC, gerM, spmA, spmB, and yqfC were missing in the genomes of all members of the family Planococcaceae (Table S4), to which $L$. sphaericus was recently reassigned (58). These observations showed that, rather than being "the worst offender" in terms of genome content, $L$. sphaericus could be a valuable model organism to study the basics of the engulfment process.

The expansion of the initial genome set to include two spore-forming members of Erysipelotrichia, C. innocuum and E. ramosum, provided a window into an even greater loss of core sporulation genes (Table S4). In these organisms, sporulation appears to be less frequent than in members of other lineages, making them somewhat similar to oligo-sporulating mutants of $B$. subtilis. Nevertheless, the ability of $C$. innocuum and $E$. ramosum to form spores in the absence of numerous core genes (Table S4) makes them useful model organisms for study minimal requirements for sporulation among the Firmicutes.

The conservation of core sporulation genes in Bacilli and Clostridia suggested that the ability to form spores was a common ancient feature of the Firmicutes $(2,4)$. Although the scenarios of the origin of Gram-negative (diderm) bacteria from spore-forming Gram-positive (monoderm) ones $(130,131)$ do not seem to be supported by phylogenetic trees of universal genes $(120,132$, 133), understanding the fundamental mechanisms of sporulation and clarifying the origin of sporeformers remains a major goal in microbiology. Here, we used an expanded collection of the firmicute genomes to trace the phylogeny of the core sporulation genes within this phylum. Despite certain variability in the branching order (Supplemental file 2), proteins coming from the members of the same phylum typically formed well-supported clades. This was particularly clearly demonstrated by a comparison of the trees built from concatenated alignments of sporulation proteins and ribosomal proteins (Figure 3). We and others have previously demonstrated that such a ribosomal proteins-based tree is closely similar to the 16S rRNA gene-based tree and faithfully represents organismal phylogeny of the Firmicutes $(28,49,134)$. The remarkable congruence of these trees strongly suggested vertical inheritance of the sporulation genes from a common ancestor of all Firmicutes. Further, in the Spo0A tree (Figure S3), proteins from nonspore-formers often grouped together with Spo0As from spore-formers of the same bacillar or clostridial family, suggesting that SpoOA-encoding non-spore-formers lost the ability to form spores, rather than acquired their spo0A genes. Accordingly, non-spore-forming Spo0A ${ }^{+}$bacteria typically retain many more sporulation genes than $\mathrm{SpoOA}^{-}$non-spore-formers (Figure 1). These 
observations lend further support to the hypothesis that the ability to form (endo)spores was an ancestral feature of the Firmicutes that emerged in this phylum at the early stages of bacterial evolution $(4,32)$. This ability remains a unique feature of some Firmicutes, distinguishing them from all other bacterial lineages. Evolution of Firmicutes from their spore-forming last common ancestor involved extensive, but in most cases, incomplete loss of sporulation genes in those lineages that lost the sporulation capacity. Subsets of the sporulation genes were also lost in several lineages of spore-formers. Experimental study of sporulation in these organisms with reduced complements of sporulation genes can be expected to yield further insight into the minimal biochemical requirements for sporulation.

Despite the substantial progress made in the past several years, a large fraction of sporulation genes remain uncharacterized. For some genes, this is reflected in their $y$-names, but many genes with specific names have been characterized only with respect to the timing of their expression, as being controlled by sporulation-specific sigma factors, or as encoding proteins of the spore coat, whereas their biochemical activities remain obscure. For example, spore maturation proteins SpmA and SpmB, which are conserved in all spore-formers except for the members of Planococcaceae (Table S6), participate in spore core dehydration and are required for heat resistance of spores $(135,136)$. However, the biochemical mechanisms of spore dehydration by SpmA and SpmB remain unknown. We hope that phylogenetic profiles of the key sporulation proteins derived in this work (Table S2) help identifying priority targets for experimental research and allow the selection of suitable model organisms for such studies.

\section{MATERIALS AND METHODS}

Genome coverage. The list of Firmicute genomes used in this work was taken from the recent release of the COG database (39). The COG genome set includes complete genomes of 175 members of the Firmicutes: 73 members of the class Bacilli, representing 59 different genera; 79 members of the class Clostridia, representing 76 genera; 10 members of the class Negativicutes, 9 members of the class Tissierellia, 2 members of the class Erysipelotrichia, 1 member of the class Limnochordia, and an additional genome of "Ndongobacter massiliensis", which remained unclassified at the time of this work (137). Most genera were represented by a single genome, except for five members each of Bacillus spp. and Streptococcus spp., four members each of Clostridium spp. and Lactobacillus spp., three Staphylococcus spp., and two Listeria spp. The list 
of these organisms is available in Table S1 in the Supplemental material and also on the NCBI website https://ftp.ncbi.nih.gov/pub/COG/COG2020/data/cog-20.org.csv. Examination of the available publications allowed extracting sporulation characteristics for 173 out of these 175 organisms. For 74 of these, the ability to form spores was documented in the descriptions of the respective species (Table S1). Ninety-nine organisms were non-spore-forming, based on the explicit descriptions of the respective strains and/or their lineages (such as the order Lactobacillales and families Acidaminococcaceae, Halanaerobiaceae, Listeriaceae, Peptoniphilaceae, Selenomonadaceae, Staphylococcaceae, and Veillonellaceae). For two organisms, the sporulation status remained unknown, as their (in)ability to form spores had not been properly documented and the respective lineages included both spore-formers and asporogens (Table S1).

Since both members of the Erysipelotrichia included in the COGs were asporogenic, the genomic list for this class was supplemented with five recently sequenced complete genomes: two from spore-forming members of the genus Erysipelatoclostridium, [Clostridium] innocuum ATCC 14501 (Genbank accession CP048838.1) and Erysipelatoclostridium ramosum DSM 1402

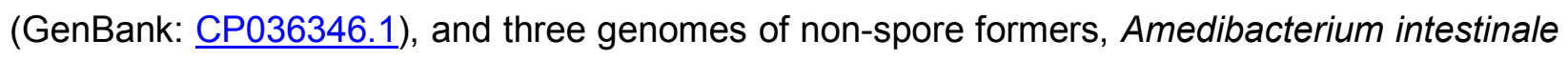
(GenBank: AP019711.1), Faecalibaculum rodentium (GenBank: CP011391.1), and Intestinibaculum porci (GenBank: AP019309.1). This brought the total set to 180 genomes, 76 of which came from proven spore-formers (Table S1).

Protein selection. The list of sporulation proteins analyzed in this work was based on the lists of genes that were previously demonstrated to play a role in sporulation of $B$. subtilis and/or were regulated either by Spo0A or by sporulation-specific sigma subunits SigE, SigF, SigG, or SigK (2, $7,12,13,15-18,33,34,115,138)$, as documented in the SubtiWiki database (38).

The selected genes were matched against the COG database (39) and the patterns of presence or absence of the respective COGs in the selected organisms were recorded in Table S2 in the Supplemental materials (in some cases, a single COG included several paralogous sporulation genes). The resulting set consisted of 237 protein families (COGs), which included 112 COGs for widely conserved genes from the previous COG releases, such as ald (spoVN), ftsH (spoVK), ftsl (spoVE), ftsK (spollIE), spmA, spmB, spoVG, spoVS, and so on (Table S2). That list was expanded by the addition of 125 sporulation COGs that were made available in the 
recent release of the COG database (39). While this list mostly contained genes (proteins) characterized in B. subtilis, it also included clostridial forms of SpolIQ (COG5833) and small acidsoluble spore protein (SASP, COG5864), as well as the Amidase_6 domain (COG5877). The widely conserved sporulation genes (proteins) were assigned to one of the following eight functional groups: 1) Sporulation onset and checkpoints (22 genes); 2) Spo0A-regulated genes (9 genes); 3) Engulfment (12 genes); 4) Spore maturation, SigF- or SigG-regulated forespore expression (19 genes); 5) Spore maturation, SigE- or SigK-regulated mother cell expression (25 genes); 6) Spore cortex synthesis (9 genes; 7) Spore coat and crust proteins (8 genes), and 8) Germination proteins (8 genes) (see Table S2). Two more groups included sporulation genes conserved in Bacilli (51 genes) and narrowly represented genes (74 genes) (Table S2). This selection was based on the previous studies $(2,15,16,28,32,36,37)$ and the data from the SubtiWiki database (http://subtiwiki.uni-goettingen.de/) (38).

Verification of the COG profiles. The patterns of presence-absence of the sporulation genes in the 180 analyzed genomes were extracted from the COG master file cog-20.cog.csv, which is available in the https://ftp.ncbi.nih.gov/pub/COG/COG2020/data/ folder, matched against the assembly numbers listed in Table S1, and the counts of each gene in the 76 spore-former genomes were calculated, ignoring the paralogs. The cases of apparently essential sporulation proteins missing in certain spore-former genomes were verified using a recent version of the TBLASTn program (55) that allows selection of specific target organisms (or lineages) based on their entries in the NCBI Taxonomy database (56). Proteins from either $B$. subtilis reference set or closely related organisms (same genus or family) were used as queries. The resulting BLAST hits (cut-off E-value, 0.1) were verified using the CD-search (139) and compared against the protein sets in GenBank and RefSeq databases. Confirmed sporulation genes were used to correct the COG profiles. Newly translated sporulation ORFs were reported to GenBank and/or RefSeq.

Phylogenetic analysis. Unless indicated otherwise, protein sequences for phylogenetic analyses were aligned with MUSCLE v5 (140), and the maximum-likelihood trees were inferred using a locally installed version of IQ-TREE 2 (118) with the best-fit model automatically selected by ModelFinder (119) and branch support calculated using Bayesian-like approximate likelihood test (141). For each COG that included paralogs, a consensus was produced as described in (145); a pairwise similarity score between each sequence and the consensus sequence was 
calculated using the BLOSUM62 matrix (with the value of -4 assigned to a gap in the sequence, matching a non-gap character of the consensus sequence); the paralog with the maximum score was used in the alignment. Individual phylogenetic trees, constructed using IQ-TREE 2 (118), are presented in Supplementary file 2.

For the phylogenetic analysis of Spo0A, the alignment included sequences from all 118 Spo0A-encoding organisms in the analyzed set: 76 spore-formers, 40 non-spore-formers, and 2 organisms with uncertain sporulation status (Table S1). The MUSCLE alignment was edited to remove variable-length linkers between the REC and DNA-binding domains (corresponding to the residues 131-148 in the Spo0A of $B$. subtilis), which left 225 informative sites. The maximumlikelihood tree of Spo0A (Figure S3) was constructed with IQ-TREE 2 (118) using the LG+R6 substitution model.

The SpoIVCA tree (Fig. S4B) was constructed from an alignment of 1,220 SpolVCA-like sitespecific DNA recombinases/integrases from bacteria and phages that were identified in a PSIBLAST (55) search. The alignment contained 476 informative positions. The approximately maximum-likelihood tree was built with FastTree 2 (142) using "-gamma" option to rescale the branch lengths and the WAG (143) model of amino acid evolution.

For phylogenetic analysis of sporulation, alignments of 41 core sporulation proteins from 76 spore-formers were constructed [the 40 genes marked in Table 1 with the addition of YmfB (COG0740), a SASP-degrading paralog of CIpP (144)]. The few missing proteins (Table S2) were replaced with the respective sequences from closely related organisms (the same genus, where available). For the overall phylogenetic tree of sporulation core, these 41 alignments were concatenated, resulting in an alignment with a total of 10,129 informative sites, and the maximumlikelihood tree (Figure 3A) was built using IQ-TREE 2 (118) with the LG+F+R9 substitution model. The ribosomal proteins-based tree (Figure $3 \mathrm{~B}$ ) was built from a concatenated sequence alignment of 54 ribosomal proteins from 76 spore-formers with IQ-TREE 2 and the LG+R8 substitution model.

\section{ACKNOWLEDGEMENTS}

This study was supported by the Intramural Research Program of the U.S. National Library of Medicine at the National Institutes of Health. 


\section{REFERENCES}

1. Errington J. 1993. Bacillus subtilis sporulation: regulation of gene expression and control of morphogenesis. Microbiol Rev 57:1-33.

2. de Hoon MJ, Eichenberger P, Vitkup D. 2010. Hierarchical evolution of the bacterial sporulation network. Curr Biol 20:R735-745.

3. Paredes-Sabja D, Setlow P, Sarker MR. 2011. Germination of spores of Bacillales and Clostridiales species: mechanisms and proteins involved. Trends Microbiol 19:85-94.

4. Galperin MY. 2013. Genome diversity of spore-forming Firmicutes. Microbiol Spectr 1:TBS-0015-2012.

5. Setlow P. 2014. Spore resistance properties. Microbiol Spectr 2:TBS-0003-2012.

6. Tan IS, Ramamurthi KS. 2014. Spore formation in Bacillus subtilis. Environ Microbiol Rep 6:212-225.

7. Driks A, Eichenberger P. 2016. The spore coat. Microbiol Spectr 4:TBS-0023-2016.

8. Khanna K, Lopez-Garrido J, Pogliano K. 2020. Shaping an endospore: architectural transformations during Bacillus subtilis sporulation. Annu Rev Microbiol 74:361-386.

9. Traag BA, Driks A, Stragier P, Bitter W, Broussard G, Hatfull G, Chu F, Adams KN, Ramakrishnan L, Losick R. 2010. Do mycobacteria produce endospores? Proc Natl Acad Sci USA 107:878-881.

10. Beskrovnaya P, Fakih D, Morneau I, Hashimi A, Bello DG, Xing S, Nanci A, Huan T, Tocheva El. 2020. No endospore formation confirmed in members of the phylum Proteobacteria. Appl Environ Microbiol 87:e02312-02320.

11. Paulson JN, Williams BL, Hehnly C, Mishra N, Sinnar SA, Zhang L, Ssentongo P, Mbabazi-Kabachelor E, Wijetunge DSS, von Bredow B, Mulondo R, Kiwanuka J, Bajunirwe F, Bazira J, Bebell LM, Burgoine K, Couto-Rodriguez M, Ericson JE, Erickson T, Ferrari M, Gladstone M, Guo C, Haran M, Hornig M, Isaacs AM, Kaaya BN, Kangere SM, Kulkarni AV, Kumbakumba E, Li X, Limbrick DD, Jr., Magombe J, Morton SU, Mugamba J, Ng J, Olupot-Olupot P, Onen J, Peterson MR, Roy F, Sheldon K, Townsend R, Weeks AD, Whalen AJ, Quackenbush J, Ssenyonga P, Galperin MY, Almeida M, Atkins H, Warf BC, Lipkin WI, et al. 2020. Paenibacillus infection with frequent viral coinfection contributes to postinfectious hydrocephalus in Ugandan infants. Sci Transl Med 12: eaba0565.

12. Stragier P, Losick R. 1996. Molecular genetics of sporulation in Bacillus subtilis. Annu Rev Genet 30:297-241.

13. Piggot PJ, Losick R. 2002. Sporulation genes and intercompartmental regulation, $p$ 483-518. In Sonenshein AL, Hoch JA, Losick R (ed), Bacillus subtilis and its closest relatives: from genes to cells. ASM Press, Washington, D.C.

14. Piggot PJ, Hilbert DW. 2004. Sporulation of Bacillus subtilis. Curr Opin Microbiol 7:579586.

15. Eichenberger P, Jensen ST, Conlon EM, van Ooij C, Silvaggi J, Gonzalez-Pastor JE, Fujita M, Ben-Yehuda S, Stragier P, Liu JS, Losick R. 2003. The $s^{E}$ regulon and the identification of additional sporulation genes in Bacillus subtilis. J Mol Biol 327:945972. 
16. Eichenberger P, Fujita M, Jensen ST, Conlon EM, Rudner DZ, Wang ST, Ferguson C, Haga K, Sato T, Liu JS, Losick R. 2004. The program of gene transcription for a single differentiating cell type during sporulation in Bacillus subtilis. PLoS Biol 2:e328.

17. Steil L, Serrano M, Henriques AO, Völker U. 2005. Genome-wide analysis of temporally regulated and compartment-specific gene expression in sporulating cells of Bacillus subtilis. Microbiology 151:399-420.

18. Wang ST, Setlow B, Conlon EM, Lyon JL, Imamura D, Sato T, Setlow P, Losick R, Eichenberger P. 2006. The forespore line of gene expression in Bacillus subtilis. J Mol Biol 358:16-37.

19. Veening JW, Murray H, Errington J. 2009. A mechanism for cell cycle regulation of sporulation initiation in Bacillus subtilis. Genes Dev 23:1959-1970.

20. Meeske AJ, Rodrigues CD, Brady J, Lim HC, Bernhardt TG, Rudner DZ. 2016. Highthroughput genetic screens identify a large and diverse collection of new sporulation genes in Bacillus subtilis. PLoS Biol 14:e1002341.

21. Liu H, Bergman NH, Thomason B, Shallom S, Hazen A, Crossno J, Rasko DA, Ravel J, Read TD, Peterson SN, Yates J, 3rd, Hanna PC. 2004. Formation and composition of the Bacillus anthracis endospore. J Bacteriol 186:164-178.

22. Lawley TD, Croucher NJ, Yu L, Clare S, Sebaihia M, Goulding D, Pickard DJ, Parkhill J, Choudhary J, Dougan G. 2009. Proteomic and genomic characterization of highly infectious Clostridium difficile 630 spores. J Bacteriol 191:5377-5386.

23. Zheng L, Abhyankar W, Ouwerling N, Dekker HL, van Veen H, van der Wel NN, Roseboom W, de Koning LJ, Brul S, de Koster CG. 2016. Bacillus subtilis spore inner membrane proteome. J Proteome Res 15:585-594.

24. Alsaker KV, Papoutsakis ET. 2005. Transcriptional program of early sporulation and stationary-phase events in Clostridium acetobutylicum. J Bacteriol 187:7103-7118.

25. Paredes CJ, Alsaker KV, Papoutsakis ET. 2005. A comparative genomic view of clostridial sporulation and physiology. Nat Rev Microbiol 3:969-978.

26. Jones SW, Paredes CJ, Tracy B, Cheng N, Sillers R, Senger RS, Papoutsakis ET. 2008. The transcriptional program underlying the physiology of clostridial sporulation. Genome Biol 9:R114.

27. Shen A, Edwards AN, Sarker MR, Paredes-Sabja D. 2019. Sporulation and germination in clostridial pathogens. Microbiol Spectr 7:GPP3-0017-2018.

28. Yutin N, Galperin MY. 2013. A genomic update on clostridial phylogeny: Gram-negative spore formers and other misplaced clostridia. Environ Microbiol 15:2631-2641.

29. Watanabe M, Kojima H, Fukui M. 2015. Limnochorda pilosa gen. nov., sp. nov., a moderately thermophilic, facultatively anaerobic, pleomorphic bacterium and proposal of Limnochordaceae fam. nov., Limnochordales ord. nov. and Limnochordia classis nov. in the phylum Firmicutes. Int J Syst Evol Microbiol 65:2378-2384.

30. Lawson PA. 2019. Tissierella, p 1-12, Bergey's Manual of Systematics of Archaea and Bacteria. John Wiley \& Sons, New York.

31. Oren A. 2014. The order Halanaerobiales, and the families Halanaerobiaceae and Halobacteroidaceae, p 153-177. In Rosenberg E, DeLong EF, Lory S, Stackebrandt E, Thompson F (ed), The Prokaryotes Firmicutes and Tenericutes 4th Edition. SpringerVerlag, Berlin Heidelberg. 
32. Galperin MY, Mekhedov SL, Puigbo P, Smirnov S, Wolf YI, Rigden DJ. 2012. Genomic determinants of sporulation in Bacilli and Clostridia: towards the minimal set of sporulation-specific genes. Environ Microbiol 14:2870-2890.

33. Onyenwoke RU, Brill JA, Farahi K, Wiegel J. 2004. Sporulation genes in members of the low G+C Gram-type-positive phylogenetic branch (Firmicutes). Arch Microbiol 182:182-192.

34. Stragier P. 2002. A gene odyssey: Exploring the genomes of endospore-forming bacteria, p 519-526. In Sonenshein AL, Hoch JA, Losick R (ed), Bacillus subtilis and its closest relatives: from genes to cells. ASM Press, Washington, D.C.

35. Wu M, Ren Q, Durkin AS, Daugherty SC, Brinkac LM, Dodson RJ, Madupu R, Sullivan SA, Kolonay JF, Haft DH, Nelson WC, Tallon LJ, Jones KM, Ulrich LE, Gonzalez JM, Zhulin IB, Robb FT, Eisen JA. 2005. Life in hot carbon monoxide: the complete genome sequence of Carboxydothermus hydrogenoformans Z-2901. PLoS Genet 1:e65.

36. Abecasis AB, Serrano M, Alves R, Quintais L, Pereira-Leal JB, Henriques AO. 2013. A genomic signature and the identification of new sporulation genes. J, Bacteriol, 195:2101-2115.

37. Ramos-Silva P, Serrano M, Henriques AO. 2019. From root to tips: sporulation evolution and specialization in Bacillus subtilis and the intestinal pathogen Clostridioides difficile. Mol Biol Evol 36:2714-2736.

38. Pedreira T, Elfmann C, Stülke J. 2022. The current state of SubtiWiki, the database for the model organism Bacillus subtilis. Nucleic Acids Res 50:gkab943.

39. Galperin MY, Wolf YI, Makarova KS, Vera Alvarez R, Landsman D, Koonin EV. 2021. COG database update: Focus on microbial diversity, model organisms, and widespread pathogens. Nucleic Acids Res 49:D274-D281.

40. Natale DA, Galperin MY, Tatusov RL, Koonin EV. 2000. Using the COG database to improve gene recognition in complete genomes. Genetica 108:9-17.

41. Tatusov RL, Koonin EV, Lipman DJ. 1997. A genomic perspective on protein families. Science 278:631-637.

42. Galperin MY, Makarova KS, Wolf YI, Koonin EV. 2015. Expanded microbial genome coverage and improved protein family annotation in the COG database. Nucleic Acids Res 43:D261-D269.

43. Sokolova T, Hanel J, Onyenwoke RU, Reysenbach AL, Banta A, Geyer R, Gonzalez JM, Whitman WB, Wiegel J. 2007. Novel chemolithotrophic, thermophilic, anaerobic bacteria Thermolithobacter ferrireducens gen. nov., sp. nov. and Thermolithobacter carboxydivorans sp. nov. Extremophiles 11:145-157.

44. Neupane S, Ghosh A, Gunther S, Martin K, Zurek L. 2020. Culicoidibacter larvae gen. nov., sp. nov., from the gastrointestinal tract of the biting midge (Culicoides sonorensis) larva, belongs to a novel lineage Culicoidibacteraceae fam. nov., Culicoidibacterales ord. nov. and Culicoidibacteria classis nov. of the phylum Firmicutes. Int J Syst Evol Microbiol 70:6482-6490.

45. Haft DH, DiCuccio M, Badretdin A, Brover V, Chetvernin V, O'Neill K, Li W, Chitsaz F, Derbyshire MK, Gonzales NR, Gwadz M, Lu F, Marchler GH, Song JS, Thanki N, Yamashita RA, Zheng C, Thibaud-Nissen F, Geer LY, Marchler-Bauer A, Pruitt KD. 
2018. RefSeq: an update on prokaryotic genome annotation and curation. Nucleic Acids Res 46:D851-D860.

46. Smith LD, King E. 1962. Clostridium innocuum, sp. n., a spore-forming anaerobe isolated from human infections. J Bacteriol 83:938-939.

47. Holdeman LV, Cato EP, Moore WEC. 1971. Clostridium ramosum (Vuillemin) comb. nov.: emended description and proposed neotype strain. Int J Syst Evol Microbiol 21:3539.

48. Karnachuk OV, Frank YA, Lukina AP, Kadnikov VV, Beletsky AV, Mardanov AV, Ravin NV. 2019. Domestication of previously uncultivated Candidatus Desulforudis audaxviator from a deep aquifer in Siberia sheds light on its physiology and evolution. ISME J 13:1947-1959.

49. Galperin MY, Brover V, Tolstoy I, Yutin N. 2016. Phylogenomic analysis of the family Peptostreptococcaceae (Clostridium cluster XI) and proposal for reclassification of Clostridium litorale (Fendrich et al. 1991) and Eubacterium acidaminophilum (Zindel et al. 1989) as Peptoclostridium litorale gen. nov. comb. nov. and Peptoclostridium acidaminophilum comb. nov. Int J Syst Evol Microbiol 66:5506-5513.

50. Poehlein A, Yutin N, Daniel R, Galperin MY. 2017. Proposal for the reclassification of obligately purine-fermenting bacteria Clostridium acidurici (Barker 1938) and Clostridium purinilyticum (Durre et al. 1981) as Gottschalkia acidurici gen. nov. comb. nov. and Gottschalkia purinilytica comb. nov. and of Eubacterium angustum (Beuscher and Andreesen 1985) as Andreesenia angusta gen. nov. comb. nov. in the family Gottschalkiaceae fam. nov. Int J Syst Evol Microbiol 67:2711-2719.

51. Davidson P, Eutsey R, Redler B, Hiller NL, Laub MT, Durand D. 2018. Flexibility and constraint: Evolutionary remodeling of the sporulation initiation pathway in Firmicutes. PLoS Genet 14:e1007470.

52. Orsburn BC, Melville SB, Popham DL. 2010. EtfA catalyses the formation of dipicolinic acid in Clostridium perfringens. Mol Microbiol 75:178-186.

53. Galperin MY, Wolf YI, Garushyants SK, Vera Alvarez R, Koonin EV. 2021. Nonessential ribosomal proteins in bacteria and archaea identified using Clusters of Orthologous Genes. J Bacteriol 203:e00058-00021.

54. Boratyn GM, Schaffer AA, Agarwala R, Altschul SF, Lipman DJ, Madden TL. 2012. Domain enhanced lookup time accelerated BLAST. Biol Direct 7:12.

55. Altschul SF, Madden TL, Schaffer AA, Zhang J, Zheng Z, Miller W, Lipman DJ. 1997. Gapped BLAST and PSI-BLAST - A new generation of protein database search programs. Nucl Acids Res 25:3389-3402.

56. Schoch CL, Ciufo S, Domrachev M, Hotton CL, Kannan S, Khovanskaya R, Leipe D, McVeigh R, O'Neill K, Robbertse B, Sharma S, Soussov V, Sullivan JP, Sun L, Turner S, Karsch-Mizrachi I. 2020. NCBI Taxonomy: a comprehensive update on curation, resources and tools. Database (Oxford) 2020: baaa062.

57. Yaakop AS, Chan KG, Ee R, Kahar UM, Kon WC, Goh KM. 2015. Isolation of Jeotgalibacillus malaysiensis sp. nov. from a sandy beach, and emended description of the genus Jeotgalibacillus. Int J Syst Evol Microbiol 65:2215-2221.

58. Gupta RS, Patel S. 2019. Robust demarcation of the family Caryophanaceae (Planococcaceae) and its different genera including three novel genera based on phylogenomics and highly specific molecular signatures. Front Microbiol 10:2821. 
59. Davis JJ, Xia F, Overbeek RA, Olsen GJ. 2013. Genomes of the class Erysipelotrichia clarify the firmicute origin of the class Mollicutes. Int J Syst Evol Microbiol 63:2727-2741.

60. Soto-Avila L, Merce RC, Santos W, Castaneda N, Gutierrez-Rios RM. 2021. Distribution and preservation of the components of the engulfment. What is beyond representative genomes? PLoS One 16:e0246651.

61. Xiao Y, Francke C, Abee T, Wells-Bennik MH. 2011. Clostridial spore germination versus bacilli: genome mining and current insights. Food Microbiol 28:266-274.

62. Clauwers C, Lood C, Van den Bergh B, van Noort V, Michiels CW. 2017. Canonical germinant receptor is dispensable for spore germination in Clostridium botulinum group II strain NCTC 11219. Sci Rep 7:15426.

63. Al-Hinai MA, Jones SW, Papoutsakis ET. 2015. The Clostridium sporulation programs: diversity and preservation of endospore differentiation. Microbiol Mol Biol Rev 79:19-37.

64. Kroos L, Zhang B, Ichikawa H, Yu YT. 1999. Control of $\sigma$ factor activity during Bacillus subtilis sporulation. Mol Microbiol 31:1285-1294.

65. Fimlaid KA, Shen A. 2015. Diverse mechanisms regulate sporulation sigma factor activity in the Firmicutes. Curr Opin Microbiol 24:88-95.

66. Sun G, Yang M, Jiang L, Huang M. 2021. Regulation of pro- $\sigma^{\mathrm{K}}$ activation: a key checkpoint in Bacillus subtilis sporulation. Environ Microbiol 23:2366-2373.

67. Stragier P, Kunkel B, Kroos L, Losick R. 1989. Chromosomal rearrangement generating a composite gene for a developmental transcription factor. Science 243:507512.

68. Kunkel B, Losick R, Stragier P. 1990. The Bacillus subtilis gene for the development transcription factor $\sigma^{\mathrm{K}}$ is generated by excision of a dispensable DNA element containing a sporulation recombinase gene. Genes Dev 4:525-535.

69. Haraldsen JD, Sonenshein AL. 2003. Efficient sporulation in Clostridium difficile requires disruption of the $\mathrm{s}^{\mathrm{K}}$ gene. Mol Microbiol 48:811-821.

70. Serrano M, Kint N, Pereira FC, Saujet L, Boudry P, Dupuy B, Henriques AO, MartinVerstraete I. 2016. A recombination directionality factor controls the cell type-specific activation of $\sigma^{\mathrm{K}}$ and the fidelity of spore development in Clostridium difficile. PLoS Genet 12:e1006312.

71. Kim KP, Born Y, Lurz R, Eichenseher F, Zimmer M, Loessner MJ, Klumpp J. 2012. Inducible Clostridium perfringens bacteriophages PhiS9 and PhiS63: Different genome structures and a fully functional sigK intervening element. Bacteriophage 2:89-97.

72. Cohen JE, Wang R, Shen RF, Wu WW, Keller JE. 2017. Comparative pathogenomics of Clostridium tetani. PLoS One 12:e0182909.

73. Ramirez-Guadiana FH, Rodrigues CDA, Marquis KA, Campo N, Barajas-Ornelas RDC, Brock K, Marks DS, Kruse AC, Rudner DZ. 2018. Evidence that regulation of intramembrane proteolysis is mediated by substrate gating during sporulation in Bacillus subtilis. PLoS Genet 14:e1007753.

74. Patterson HM, Brannigan JA, Cutting SM, Wilson KS, Wilkinson AJ, Ab E, Diercks T, de Jong RN, Truffault V, Folkers GE, Kaptein R. 2005. The structure of bypass of forespore $\mathrm{C}$, an intercompartmental signaling factor during sporulation in Bacillus. J Biol Chem 280:36214-36220. 
75. Aung S, Shum J, Abanes-De Mello A, Broder DH, Fredlund-Gutierrez J, Chiba S, Pogliano K. 2007. Dual localization pathways for the engulfment proteins during Bacillus subtilis sporulation. Mol Microbiol 65:1534-1546.

76. Chastanet A, Losick R. 2007. Engulfment during sporulation in Bacillus subtilis is governed by a multi-protein complex containing tandemly acting autolysins. Mol Microbiol 64:139-152.

77. Crawshaw AD, Serrano M, Stanley WA, Henriques AO, Salgado PS. 2014. A mother cell-to-forespore channel: current understanding and future challenges. FEMS Microbiol Lett 358:129-136.

78. Rodrigues CD, Ramirez-Guadiana FH, Meeske AJ, Wang X, Rudner DZ. 2016. GerM is required to assemble the basal platform of the SpollIA-SpollQ transenvelope complex during sporulation in Bacillus subtilis. Mol Microbiol 102:260-273.

79. Kelly A, Salgado PS. 2019. The engulfasome in C. difficile: Variations on protein machineries. Anaerobe 60:102091.

80. Dembek M, Kelly A, Barwinska-Sendra A, Tarrant E, Stanley WA, Vollmer D, Biboy J, Gray J, Vollmer W, Salgado PS. 2018. Peptidoglycan degradation machinery in Clostridium difficile forespore engulfment. Mol Microbiol 110:390-410.

81. Ribis JW, Fimlaid KA, Shen A. 2018. Differential requirements for conserved peptidoglycan remodeling enzymes during Clostridioides difficile spore formation. Mol Microbiol 110:370-389.

82. Doan T, Marquis KA, Rudner DZ. 2005. Subcellular localization of a sporulation membrane protein is achieved through a network of interactions along and across the septum. Mol Microbiol 55:1767-1781.

83. Crawshaw AD, Serrano M, Stanley WA, Henriques AO, Salgado PS. 2014. A mother cell-to-forespore channel: current understanding and future challenges. FEMS Microbiol Lett 358:129-136.

84. Levdikov VM, Blagova EV, McFeat A, Fogg MJ, Wilson KS, Wilkinson AJ. 2012. Structure of components of an intercellular channel complex in sporulating Bacillus subtilis. Proc Natl Acad Sci USA 109:5441-5445.

85. Meisner J, Maehigashi T, Andre I, Dunham CM, Moran CP, Jr. 2012. Structure of the basal components of a bacterial transporter. Proc Natl Acad Sci USA 109:5446-5451.

86. Serrano M, Crawshaw AD, Dembek M, Monteiro JM, Pereira FC, Pinho MG, Fairweather NF, Salgado PS, Henriques AO. 2016. The SpollQ-SpollIAH complex of Clostridium difficile controls forespore engulfment and late stages of gene expression and spore morphogenesis. Mol Microbiol 100:204-228.

87. Setlow P. 1988. Small, acid-soluble spore proteins of Bacillus species: structure, synthesis, genetics, function, and degradation. Annu Rev Microbiol 42:319-338.

88. Setlow P. 2007. I will survive: DNA protection in bacterial spores. Trends Microbiol 15:172-180.

89. Mason JM, Setlow P. 1986. Essential role of small, acid-soluble spore proteins in resistance of Bacillus subtilis spores to UV light. J Bacteriol 167:174-178.

90. Moeller R, Setlow P, Reitz G, Nicholson WL. 2009. Roles of small, acid-soluble spore proteins and core water content in survival of Bacillus subtilis spores exposed to environmental solar UV radiation. Appl Environ Microbiol 75:5202-5208. 
91. Cortesao M, Fuchs FM, Commichau FM, Eichenberger P, Schuerger AC, Nicholson WL, Setlow P, Moeller R. 2019. Bacillus subtilis spore resistance to simulated Mars surface conditions. Front Microbiol 10:333.

92. Bagyan I, Setlow B, Setlow P. 1998. New small, acid-soluble proteins unique to spores of Bacillus subtilis: identification of the coding genes and regulation and function of two of these genes. J Bacteriol 180:6704-6712.

93. Vyas J, Cox J, Setlow B, Coleman WH, Setlow P. 2011. Extremely variable conservation of gamma-type small, acid-soluble proteins from spores of some species in the bacterial order Bacillales. J Bacteriol 193:1884-1892.

94. Li J, McClane BA. 2008. A novel small acid soluble protein variant is important for spore resistance of most Clostridium perfringens food poisoning isolates. PLoS Pathog 4:e1000056.

95. Wetzel D, Fischer RJ. 2015. Small acid-soluble spore proteins of Clostridium acetobutylicum are able to protect DNA in vitro and are specifically cleaved by germination protease GPR and spore protease YyaC. Microbiology (Reading) 161:20982109.

96. Fawcett P, Eichenberger P, Losick R, Youngman P. 2000. The transcriptional profile of early to middle sporulation in Bacillus subtilis. Proc Natl Acad Sci USA 97:8063-8068.

97. Asai K, Takamatsu H, Iwano M, Kodama T, Watabe K, Ogasawara N. 2001. The Bacillus subtilis yabQ gene is essential for formation of the spore cortex. Microbiology 147:919-927.

98. Permpoonpattana P, Phetcharaburanin J, Mikelsone A, Dembek M, Tan S, Brisson MC, La Ragione R, Brisson AR, Fairweather N, Hong HA, Cutting SM. 2013. Functional characterization of Clostridium difficile spore coat proteins. J Bacteriol 195:1492-1503.

99. Paredes-Sabja D, Shen A, Sorg JA. 2014. Clostridium difficile spore biology: sporulation, germination, and spore structural proteins. Trends Microbiol 22:406-416.

100. Secaira-Morocho H, Castillo JA, Driks A. 2020. Diversity and evolutionary dynamics of spore-coat proteins in spore-forming species of Bacillales. Microb Genom 6:mgen000451.

101. Wang KH, Isidro AL, Domingues L, Eskandarian HA, McKenney PT, Drew K, Grabowski P, Chua MH, Barry SN, Guan M, Bonneau R, Henriques AO, Eichenberger P. 2009. The coat morphogenetic protein SpoVID is necessary for spore encasement in Bacillus subtilis. Mol Microbiol 74:634-649.

102. de Francesco M, Jacobs JZ, Nunes F, Serrano M, McKenney PT, Chua MH, Henriques AO, Eichenberger P. 2012. Physical interaction between coat morphogenetic proteins SpoVID and CotE is necessary for spore encasement in Bacillus subtilis. J Bacteriol 194:4941-4950.

103. Putnam EE, Nock AM, Lawley TD, Shen A. 2013. SpolVA and SipL are Clostridium difficile spore morphogenetic proteins. J Bacteriol 195:1214-1225.

104. Touchette MH, Benito de la Puebla H, Ravichandran P, Shen A. 2019. SpolVA-SipL complex formation is essential for Clostridioides difficile spore assembly. J Bacteriol 201:e00042-00019. 
105. Ribis JW, Ravichandran P, Putnam EE, Pishdadian K, Shen A. 2017. The conserved spore coat protein SpoVM Is largely dispensable in Clostridium difficile spore formation. mSphere 2:e00315-00317.

106. Permpoonpattana P, Tolls EH, Nadem R, Tan S, Brisson A, Cutting SM. 2011. Surface layers of Clostridium difficile endospores. J Bacteriol 193:6461-6470.

107. Donnelly ML, Li W, Li YQ, Hinkel L, Setlow P, Shen A. 2017. A Clostridium difficilespecific, gel-forming protein required for optimal spore germination. mBio 8:e0208502016.

108. Rohlfing AE, Eckenroth BE, Forster ER, Kevorkian Y, Donnelly ML, Benito de la Puebla H, Doublie S, Shen A. 2019. The CspC pseudoprotease regulates germination of Clostridioides difficile spores in response to multiple environmental signals. PLoS Genet 15:e1008224.

109. Meaney CA, Cartman ST, McClure PJ, Minton NP. 2015. Optimal spore germination in Clostridium botulinum ATCC 3502 requires the presence of functional copies of SleB and YpeB, but not CwlJ. Anaerobe 34:86-93.

110. Sayer CV, Popham DL. 2019. YpeB dimerization may be required to stabilize SleB for effective germination of Bacillus anthracis spores. BMC Microbiol 19:169.

111. Amon JD, Yadav AK, Ramirez-Guadiana FH, Meeske AJ, Cava F, Rudner DZ. 2020. SwsB and SafA are required for CwlJ-dependent spore germination in Bacillus subtilis. J Bacteriol 202:e00668-00619.

112. Mistry J, Chuguransky S, Williams L, Qureshi M, Salazar GA, Sonnhammer ELL, Tosatto SCE, Paladin L, Raj S, Richardson LJ, Finn RD, Bateman A. 2021. Pfam: The protein families database in 2021. Nucleic Acids Res 49:D412-D419.

113. Natarajan P, Punta M, Kumar A, Yeh AP, Godzik A, Aravind L. 2015. Structure and sequence analyses of Bacteroides proteins BVU_4064 and BF1687 reveal presence of two novel predominantly-beta domains, predicted to be involved in lipid and cell surface interactions. BMC Bioinformatics 16:7.

114. Nessi C, Jedrzejas MJ, Setlow P. 1998. Structure and mechanism of action of the protease that degrades small, acid-soluble spore proteins during germination of spores of Bacillus species. J Bacteriol 180:5077-5084.

115. Molle V, Fujita M, Jensen ST, Eichenberger P, Gonzalez-Pastor JE, Liu JS, Losick R. 2003. The Spo0A regulon of Bacillus subtilis. Mol Microbiol 50:1683-1701.

116. Bate AR, Bonneau R, Eichenberger P. 2014. Bacillus subtilis systems biology: applications of -omics techniques to the study of endospore formation. Microbiol Spectr 2:TBS-0019-2013.

117. Kumar S, Stecher G, Li M, Knyaz C, Tamura K. 2018. MEGA X: Molecular Evolutionary Genetics Analysis across Computing Platforms. Mol Biol Evol 35:15471549.

118. Minh BQ, Schmidt HA, Chernomor O, Schrempf D, Woodhams MD, von Haeseler A, Lanfear R. 2020. IQ-TREE 2: New models and efficient methods for phylogenetic inference in the genomic era. Mol Biol Evol 37:1530-1534.

119. Kalyaanamoorthy S, Minh BQ, Wong TKF, von Haeseler A, Jermiin LS. 2017. ModelFinder: fast model selection for accurate phylogenetic estimates. Nat Methods 14:587-589. 
120. Yutin N, Puigbo P, Koonin EV, Wolf YI. 2012. Phylogenomics of prokaryotic ribosomal proteins. PLoS One 7:e36972.

121. Cohen O, Ashkenazy H, Belinky F, Huchon D, Pupko T. 2010. GLOOME: gain loss mapping engine. Bioinformatics 26:2914-2915.

122. Cano RJ, Borucki MK. 1995. Revival and identification of bacterial spores in 25- to 40million-year-old Dominican amber. Science 268:1060-1064.

123. Browne HP, Forster SC, Anonye BO, Kumar N, Neville BA, Stares MD, Goulding D, Lawley TD. 2016. Culturing of 'unculturable' human microbiota reveals novel taxa and extensive sporulation. Nature 533:543-546.

124. Kevbrin VV, Zhilina TN, Zavarzin GA. 1995. Physiology of the halophilic homoacetic bacterium Acetohalobium arabaticum. Microbiology (Moscow) 64:134-138.

125. Sikorski J, Lapidus A, Chertkov O, Lucas S, Copeland A, Glavina Del Rio T, Nolan M, Tice H, Cheng JF, Han C, Brambilla E, Pitluck S, Liolios K, Ivanova N, Mavromatis K, Mikhailova N, Pati A, Bruce D, Detter C, Tapia R, Goodwin L, Chen A, Palaniappan K, Land M, Hauser L, Chang YJ, Jeffries CD, Rohde M, Goker M, Spring S, Woyke T, Bristow J, Eisen JA, Markowitz V, Hugenholtz P, Kyrpides NC, Klenk HP. 2010. Complete genome sequence of Acetohalobium arabaticum type strain (Z-7288). Stand Genomic Sci 3:57-65.

126. Bosshard PP, Zbinden R, Altwegg M. 2002. Turicibacter sanguinis gen. nov., sp. nov., a novel anaerobic, Gram-positive bacterium. Int J Syst Evol Microbiol 52:1263-1266.

127. Alexander CJ, Citron DM, Brazier JS, Goldstein EJ. 1995. Identification and antimicrobial resistance patterns of clinical isolates of Clostridium clostridioforme, Clostridium innocuum, and Clostridium ramosum compared with those of clinical isolates of Clostridium perfringens. J Clin Microbiol 33:3209-3215.

128. Crum-Cianflone N. 2009. Clostridium innocuum bacteremia in a patient with acquired immunodeficiency syndrome. Am J Med Sci 337:480-482.

129. Hu X, Fan W, Han B, Liu H, Zheng D, Li Q, Dong W, Yan J, Gao M, Berry C, Yuan Z. 2008. Complete genome sequence of the mosquitocidal bacterium Bacillus sphaericus C3-41 and comparison with those of closely related Bacillus species. J Bacteriol 190:2892-2902.

130. Tocheva El, Matson EG, Morris DM, Moussavi F, Leadbetter JR, Jensen GJ. 2011. Peptidoglycan remodeling and conversion of an inner membrane into an outer membrane during sporulation. Cell 146:799-812.

131. Tocheva EI, Ortega DR, Jensen GJ. 2016. Sporulation, bacterial cell envelopes and the origin of life. Nat Rev Microbiol 14:535-542.

132. Megrian D, Taib N, Witwinowski J, Beloin C, Gribaldo S. 2020. One or two membranes? Diderm Firmicutes challenge the Gram-positive/Gram-negative divide. Mol Microbiol 113:659-671.

133. Taib N, Megrian D, Witwinowski J, Adam P, Poppleton D, Borrel G, Beloin C, Gribaldo S. 2020. Genome-wide analysis of the Firmicutes illuminates the diderm/monoderm transition. Nat Ecol Evol 4:1661-1672.

134. Teeling $\mathbf{H}$, Gloeckner FO. 2006. RibAlign: a software tool and database for eubacterial phylogeny based on concatenated ribosomal protein subunits. BMC Bioinformatics 7:66. 
135. Popham DL, Illades-Aguiar B, Setlow P. 1995. The Bacillus subtilis dacB gene, encoding penicillin-binding protein $5^{*}$, is part of a three-gene operon required for proper spore cortex synthesis and spore core dehydration. J Bacteriol 177:4721-4729.

136. Orsburn B, Sucre K, Popham DL, Melville SB. 2009. The SpmA/B and DacF proteins of Clostridium perfringens play important roles in spore heat resistance. FEMS Microbiol Lett 291:188-194.

137. Brahimi S, Cadoret F, Fournier PE, Moal V, Raoult D. 2017. 'Ndongobacter massiliensis' gen. nov., sp. nov., a new bacterial genus isolated from a human urine sample after de novo kidney transplantation. New Microbes New Infect 16:34-36.

138. Lai EM, Phadke ND, Kachman MT, Giorno R, Vazquez S, Vazquez JA, Maddock JR, Driks A. 2003. Proteomic analysis of the spore coats of Bacillus subtilis and Bacillus anthracis. J Bacteriol 185:1443-1454.

139. Lu S, Wang J, Chitsaz F, Derbyshire MK, Geer RC, Gonzales NR, Gwadz M, Hurwitz DI, Marchler GH, Song JS, Thanki N, Yamashita RA, Yang M, Zhang D, Zheng C, Lanczycki CJ, Marchler-Bauer A. 2020. CDD/SPARCLE: the conserved domain database in 2020. Nucleic Acids Res 48:D265-D268.

140. Edgar RC. 2021. MUSCLE v5 enables improved estimates of phylogenetic tree confidence by ensemble bootstrapping. bioRxiv 2021.06.20.449169.

141. Anisimova M, Gil M, Dufayard JF, Dessimoz C, Gascuel O. 2011. Survey of branch support methods demonstrates accuracy, power, and robustness of fast likelihood-based approximation schemes. Syst Biol 60:685-699.

142. Price MN, Dehal PS, Arkin AP. 2010. FastTree 2--approximately maximum-likelihood trees for large alignments. PLoS One 5:e9490.

143. Whelan S, Goldman N. 2001. A general empirical model of protein evolution derived from multiple protein families using a maximum-likelihood approach. Mol Biol Evol 18:691-699.

144. Traag BA, Pugliese A, Setlow B, Setlow P, Losick R. 2013. A conserved ClpP-like protease involved in spore outgrowth in Bacillus subtilis. Mol Microbiol 90:160-166.

145. Esterman ES, Wolf YI, Kogay R, Koonin EV, Zhaxybayeva O. 2021. Evolution of DNA packaging in gene transfer agents. Virus Evol 7:veab015. 
Table 1. Conservation of sporulation genes in spore-forming Firmicutes

\begin{tabular}{|c|c|c|}
\hline \multirow{2}{*}{$\begin{array}{l}\text { Sporulation } \\
\text { stage or regulon }\end{array}$} & \multicolumn{2}{|l|}{ Distribution of sporulation genes ${ }^{a}$} \\
\hline & Universally conserved in Firmicutes & Conserved mostly in Bacilli \\
\hline $\begin{array}{l}\text { Sporulation onset } \\
\text { and checkpoints, } \\
\text { asymmetric cell } \\
\text { division }\end{array}$ & 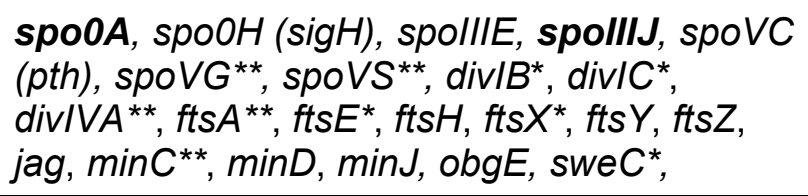 & $\begin{array}{l}\text { spo0B, spoOF, spo0E, spoVN } \\
\text { (ald), ftsL, ricA (ymcA), ricF } \\
(y l b F), \text { ric } T \text { (yaaT) }\end{array}$ \\
\hline Spo0A regulon & $\begin{array}{l}\text { sigE, sigF, sigG, spollAA, spollAB, spollE, } \\
\text { spolIGA, spoOJA (parA) }{ }^{*}, \text { spoOJB (parB), }\end{array}$ & yisK, yusE \\
\hline Engulfment & 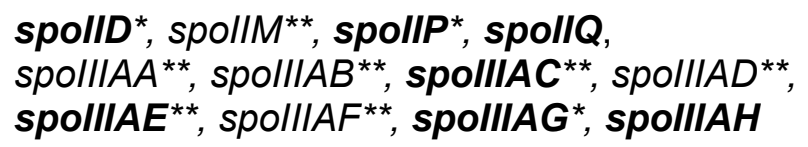 & spollB, spollIL, fisB (yunB) \\
\hline \multicolumn{3}{|c|}{ Forespore-expressed genes } \\
\hline SigF regulon & 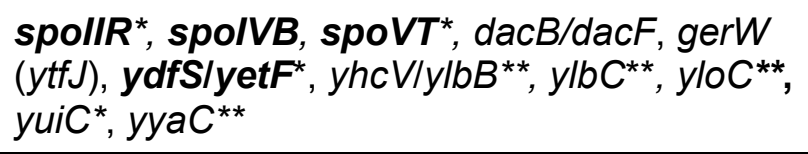 & $\begin{array}{l}\text { bofC, } r s f A, \text { fin }(y a b K), s d a, y j b A, \\
y m f J, y q h G, y w z B\end{array}$ \\
\hline SigG regulon & $\begin{array}{l}\text { spoVAC, spoVAD, spoVAEB, nfo (yqfS) }{ }^{* *} \\
\text { sspA/sspB/sspC/sspD }\end{array}$ & $\begin{array}{l}\text { spoVAA, spoVAB, spoVAF, } \\
\text { sspE, sspF, sspH, sspl, tlp, } \\
\text { yqfX }\end{array}$ \\
\hline \multicolumn{3}{|c|}{ Mother cell-expressed genes } \\
\hline SigE regulon & 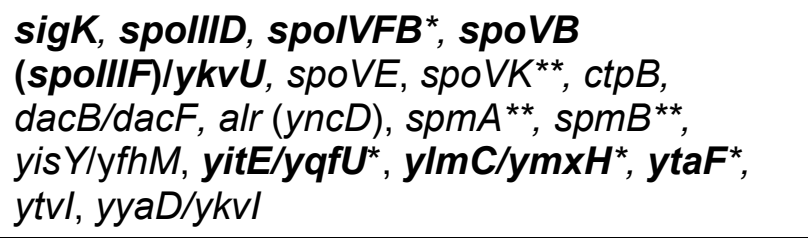 & $\begin{array}{l}\text { spolVFA, spoVM, bofA, ydcA, } \\
\text { ydcC, yhbH }\end{array}$ \\
\hline SigK regulon & $\begin{array}{l}d p a A(\operatorname{spoV} F A)^{* *}, d p a B(\text { spoVFB })^{* *}, \text { cge } D^{* *} \\
y k u D / y \operatorname{ciB} B^{* *}, y t d A^{* *}, y t I D^{* *}\end{array}$ & \\
\hline Spore cortex & $\begin{array}{l}\text { spoVD, spoVV (ylbJ), cwlC/cwlD, lytt } H^{* *} \text {, sto } A \\
\text { (spolVH), yabP, yabQ*, yqfC }{ }^{* *}, \text { yqfD }\end{array}$ & $\cot D$ \\
\hline Spore coat & $\begin{array}{l}\text { spolVA, cotJC/yjqC } C^{* *}, \cot S A, \text { gerM }{ }^{* *}, \text { lipC } \\
(y \operatorname{csK})^{* *}, \text { safA }^{* *}, \text { sleL/ydhD }{ }^{* *}, \text { yhaX }\end{array}$ & $\begin{array}{l}\text { spoVID, spoVIF, } \cot E, \operatorname{cotJ} A \\
\operatorname{cotJB}, \operatorname{cotM} / \cot P, y h j R\end{array}$ \\
\hline Germination & 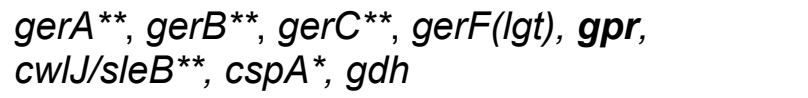 & gerD, gerE, gerQ, ypeB \\
\hline
\end{tabular}

a Distribution of the COGs that contain the indicated Bacillus subtilis sporulation genes. No distinction is made for members of the same COG. Paralogs are separated with a slash, parentheses indicate alternative names of the same gene, see Table S2 for details. Genes whose products were used to build the consensus tree (Figure 3A) are shown in bold typeface.

* Genes that are missing in one or two genomes of spore-formers (not counting the frameshifts), see Table S3 for a detailed list.

** Genes that are conserved in diverse spore-formers but missing in three or more spore-former genomes. 


\section{Legend to Figures}

\section{Figure 1. Distribution of sporulation genes in $\mathrm{SpoOA}^{+}$and $\mathrm{SpoOA}^{-}$firmicutes.}

The graphs show the numbers of widespread sporulation genes (A, out of 112), those conserved mostly in Bacilli (B, out of 51 ), and more narrowly conserved sporulation genes (C, out of 74 ) encoded in spore-formers (which are all SpoOA+, blue columns), Spo0A non-sporeformers (orange columns), and Spo0A- non-spore-formers (gray columns). The column groups represent, from left to right, all firmicutes, bacilli, clostridia, and members of the other four classes (Negativicutes, Tissierellia, Erysipelotrichia, and Limnochordia).

\section{Figure 2. Organization of skin elements in 15 firmicute genomes.}

spoIVCB genes are shown in light green, spollIC in dark green, spolVCA in orange. The organism names are followed by the GenBank genome entries and genomic coordinates of the regions between spoIVCB and spoIIIC. The numbers above the gaps indicate the distances between spoIVCB or spollIC and spoIVCA ( 1.5 kb less than the total length of the skin element). R. cellulolyticum Ccel_0434 is annotated as a single 7,394-nt pseudogene.

Figure 3. Phylogenic trees for sporulation and ribosomal proteins from Firmicutes Maximum-likelihood phylogenetic trees were built using IQ-TREE2 (118) with the LG+F+R9 substitution model from concatenated alignments of 41 core sporulation proteins $(A)$ and 54 ribosomal proteins $(B)$ encoded in the genomes of 76 spore-forming members of the Firmicutes. 
A

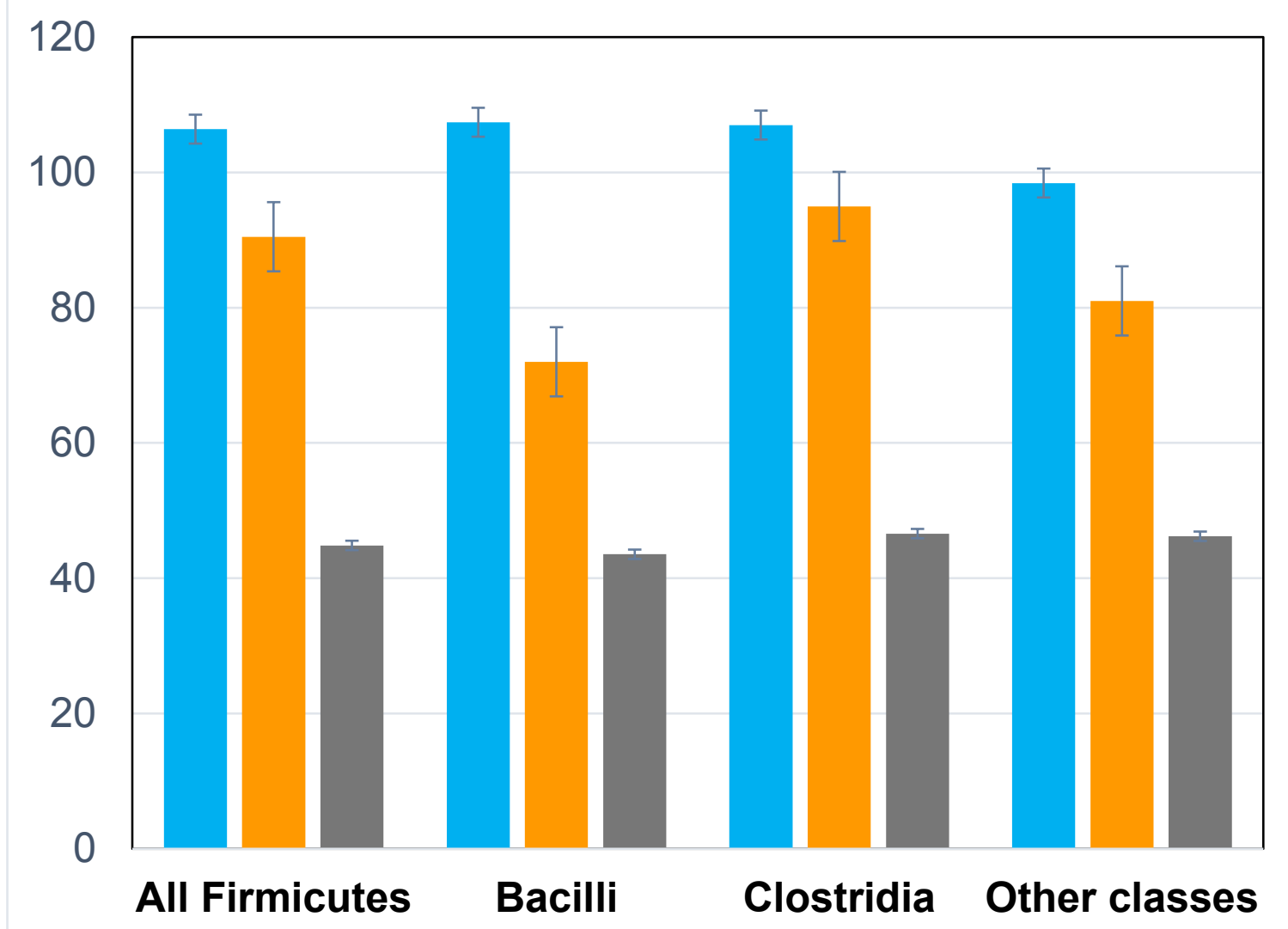

B

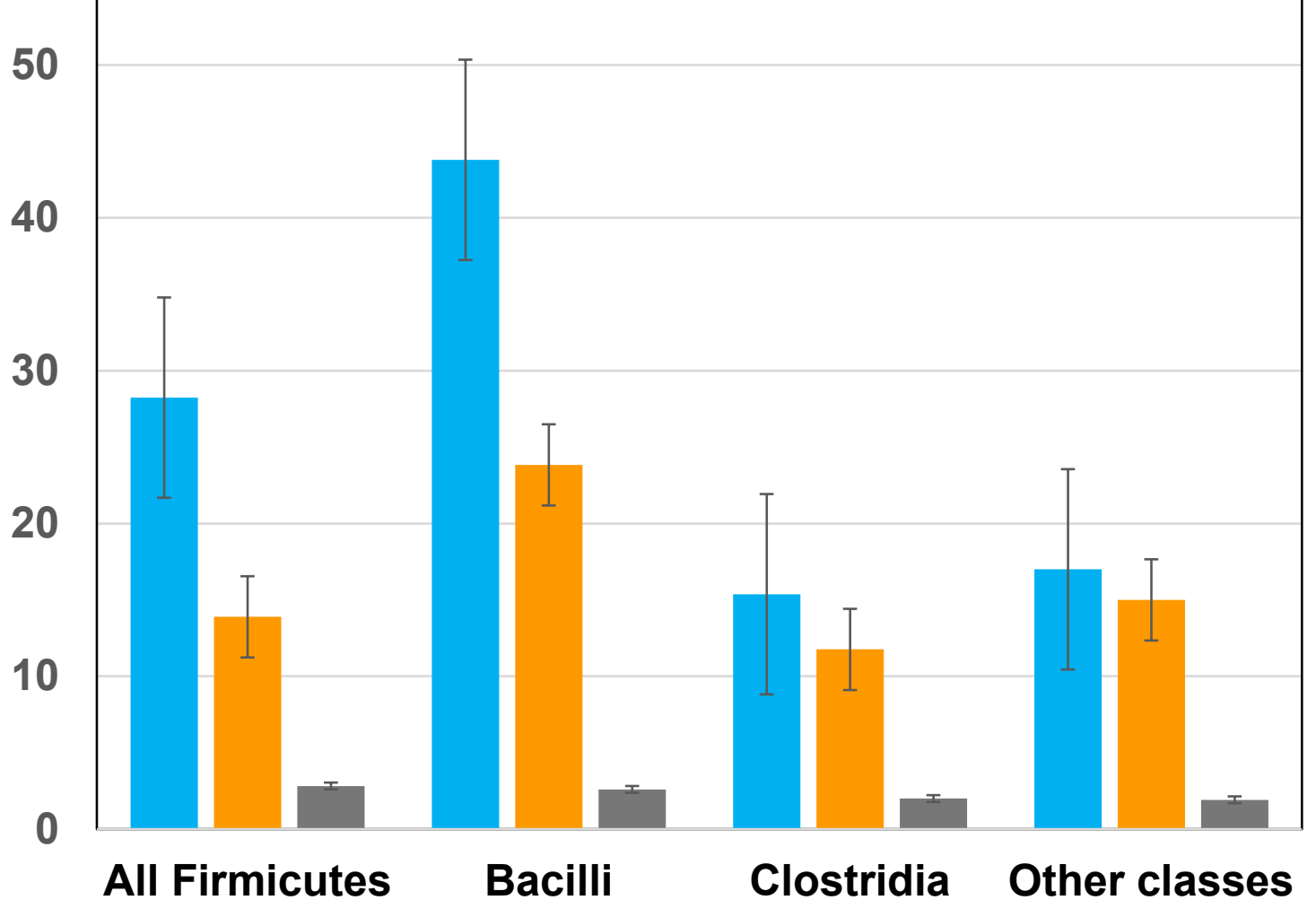




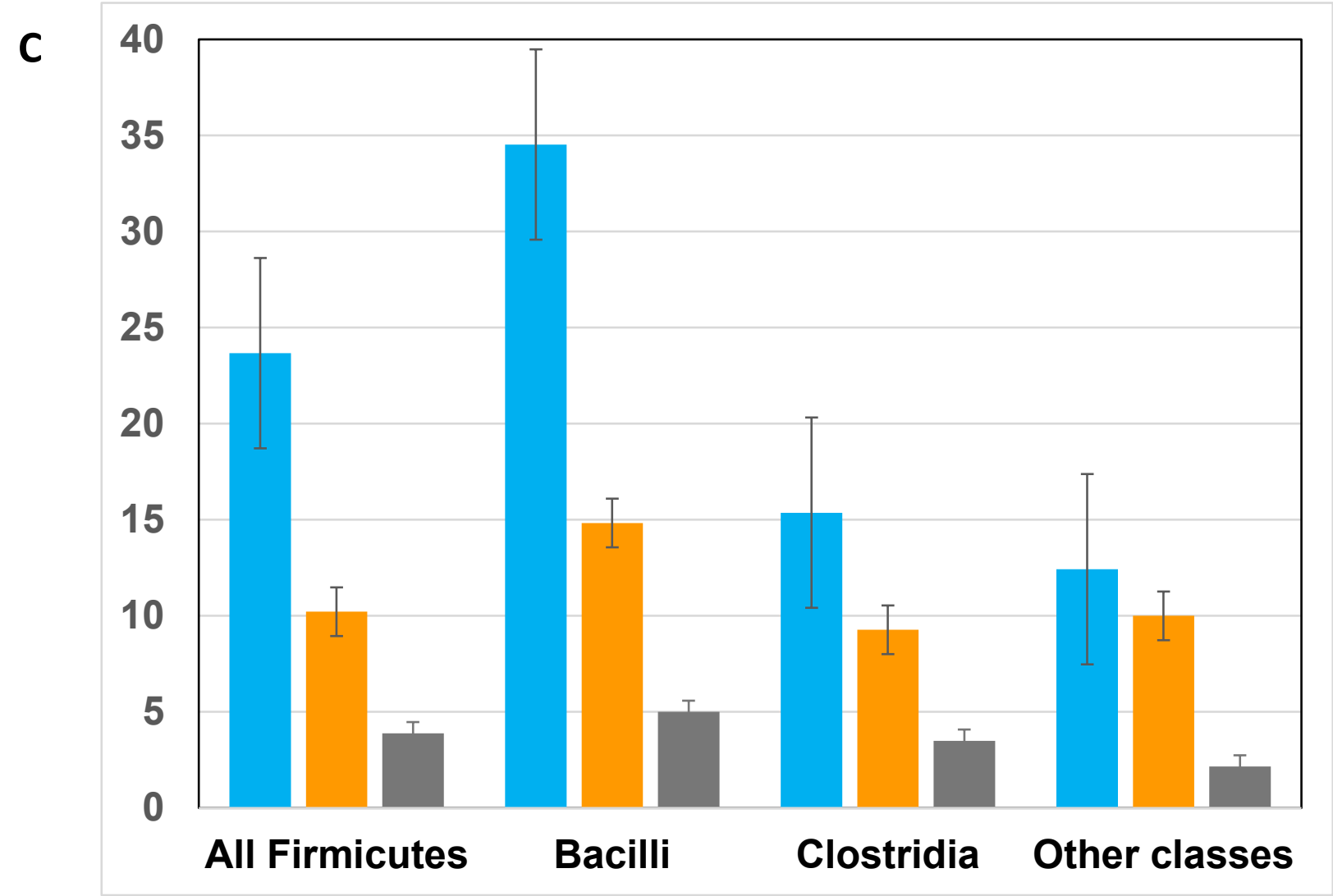

Figure 1. Distribution of sporulation genes in Spo0A ${ }^{+}$and $\mathrm{SpoOA}^{-}$firmicutes. The graphs show the numbers of widespread sporulation genes ( $A$, out of 112), those conserved mostly in Bacilli ( $B$, out of 51$)$ and more narrowly conserved sporulation genes ( $C$, out of 74 ) encoded in firmicute spore-formers (which are all Spo0A ${ }^{+}$, blue columns), SpoOA ${ }^{+}$non-spore-formers (orange columns), and Spo0 $\mathrm{A}^{-}$non-spore-formers (gray columns). The column groups represent, from left to right, all firmicutes, bacilli, clostridia, and members of the other four classes (Negativicutes, Tissierellia, Erysipelotrichia, and Limnochordia). Error bars indicate variation within each group. The source data are presented in Table S2. 
Bacillus subtilis subsp. subtilis str. 168, AL009126.3: 2,653,463-2,701,338

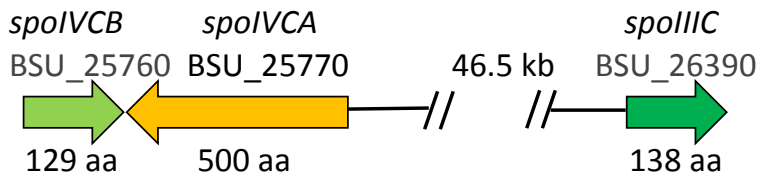

Alkaliphilus metalliredigens QYMF: CP000724.1: 2,523,887 - 2,527,436

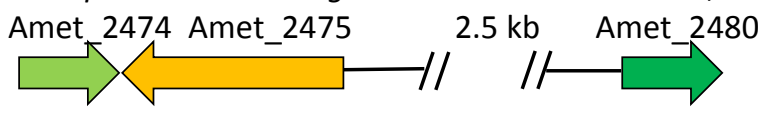

Anoxybacillus flavithermus WK1, CP000922.1: 818,174 - 834,234

Aflv_0775 $\quad 14.6 \mathrm{~kb} \quad$ Aflv_0803 Aflv_0804

bioRxiv prepr (which was not certified by peer review) is the authorfunder. This article is a US Government work. It is not subject to copyright under 17 USC

105 and is also made available for use under a CCO license.

NBRC 100599, AP008955.1: 2,017,400 - 2,026,645

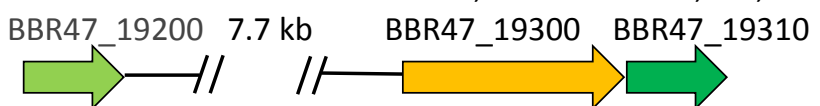

Caproiciproducens sp. NJN-50, CP035283.1: 856,703 - 868,853

EQM14_04145 EQM14_04150 10.4 kb EQM14_04205

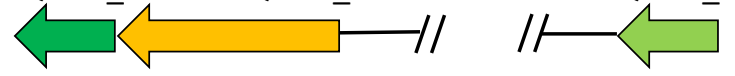

Clostridioides difficile 630, AM180355.1: 1,429,690 - 1,444,350 CD630_12300 CD630_12310 $13.1 \mathrm{~kb}$ CD630_12300

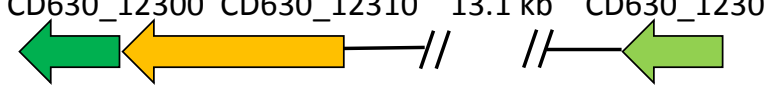

Clostridium tetani E88, AE015927.1: 1,138,064 - 1,185,018

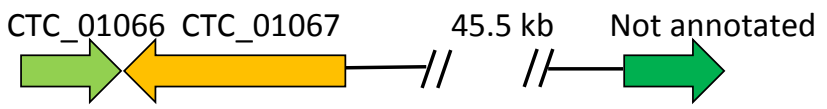

Gottschalkia acidurici 9a, CP003326.1: 1,450,043 - 1,461,566

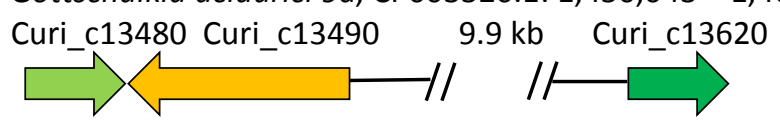

Natranaerobius thermophilus JW/NM-WN-LF, CP001034.1: 1,803,268 - 1,837,025

Nther_1737 Nther_1738 $32.2 \mathrm{~kb} \quad$ Nther_1770

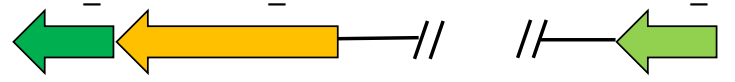

Novibacillus thermophilus SG-1, CP019699.1: 1,053,756 - 1,092,225

BOW44 $0518037.0 \mathrm{~kb} \quad$ B0W44_05430 B0W44_05435
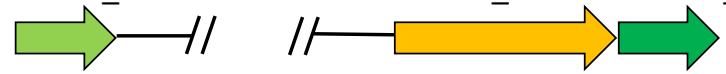

Pelotomaculum thermopropionicum SI, AP009389.1: 1,160,494 - 1,164,714

PTH_1122 $2.7 \mathrm{~kb} \quad$ PTH_1125 PTH_1126

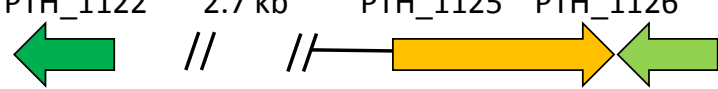

Pelosinus fermentans JBW45, CP010978.1: 2,227,052 - 2,233,164

JBW_01920 JBW_01921 $4.5 \mathrm{~kb} \quad$ Not annotated

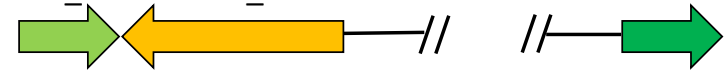

Ruminiclostridium cellulolyticum H10, CP001348.1: 498,973 - 505,588

Ccel_0434 (5.0 kb) Ccel_0440

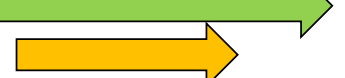

Sulfobacillus acidophilus TPY, CP002901.1: 3,074,214 - 3,076,842

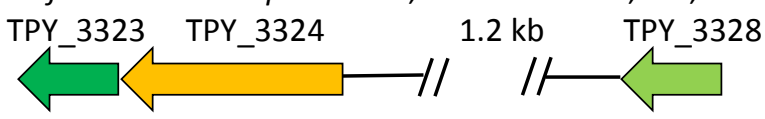

Thermacetogenium phaeum DSM 12270, CP003732.1: 1,687,787 - 1,691,412

Tph_c16780 Tph_c16790

$2.1 \mathrm{~kb}$ Tph_c16830

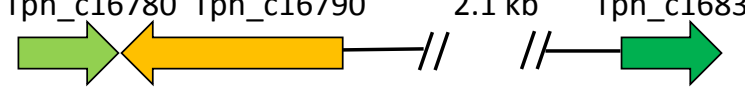

Figure 2. Organization of skin elements in $\mathbf{1 5}$ firmicute genomes.

spoIVCB genes are shown in light green, spollIC in dark green, spolVCA in orange. The organism names are followed by the GenBank genome entries and genomic coordinates of the regions between $s p o I V C B$ and spoll/C. The numbers above the gaps indicate the distances between spoIVCB or spollIC and spolVCA ( $1.5 \mathrm{~kb}$ less than the total length of the skin element). Ruminiclostridium cellulolyticum Ccel_0434 is annotated as a single 7,394-nt pseudogene. 
A

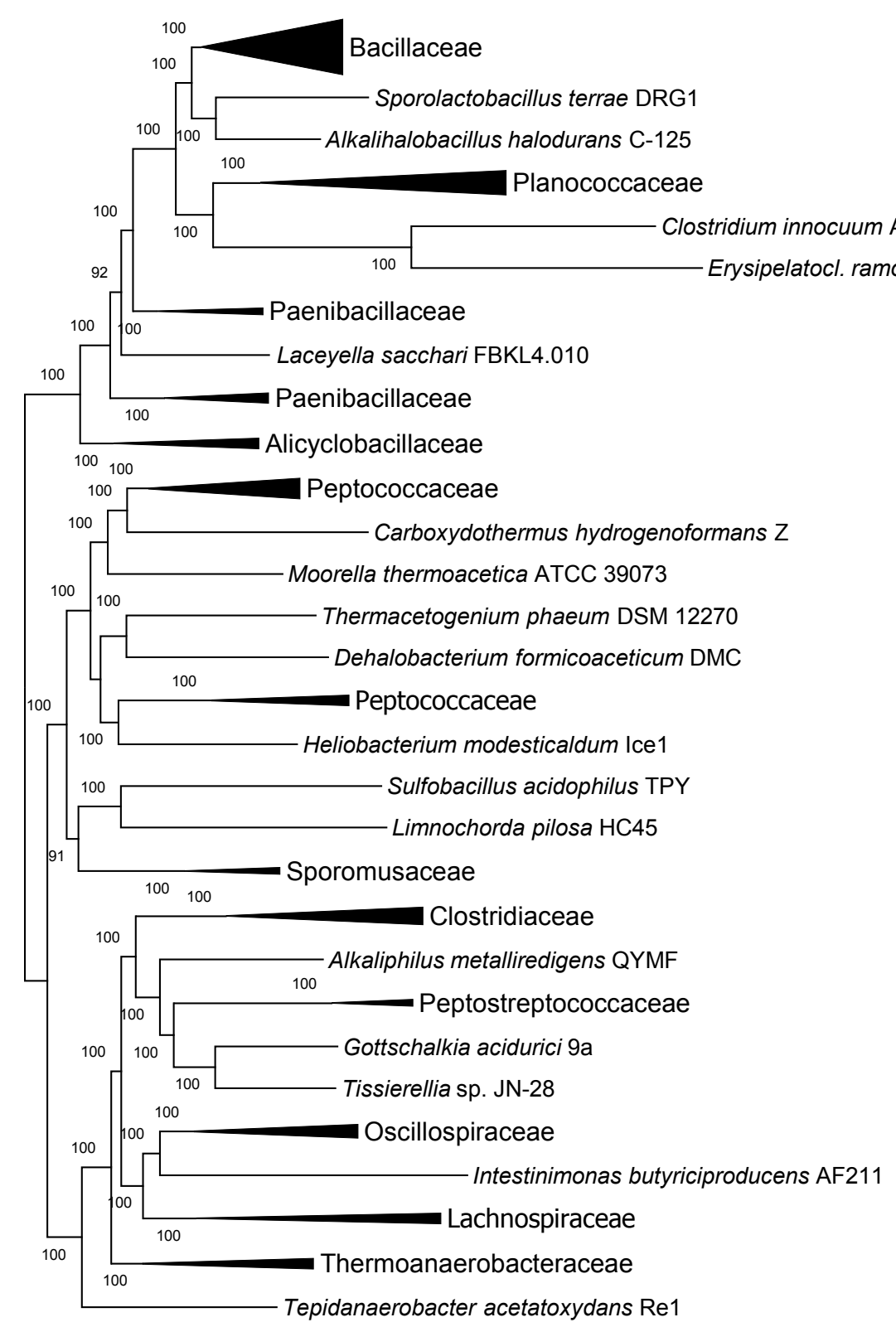

B

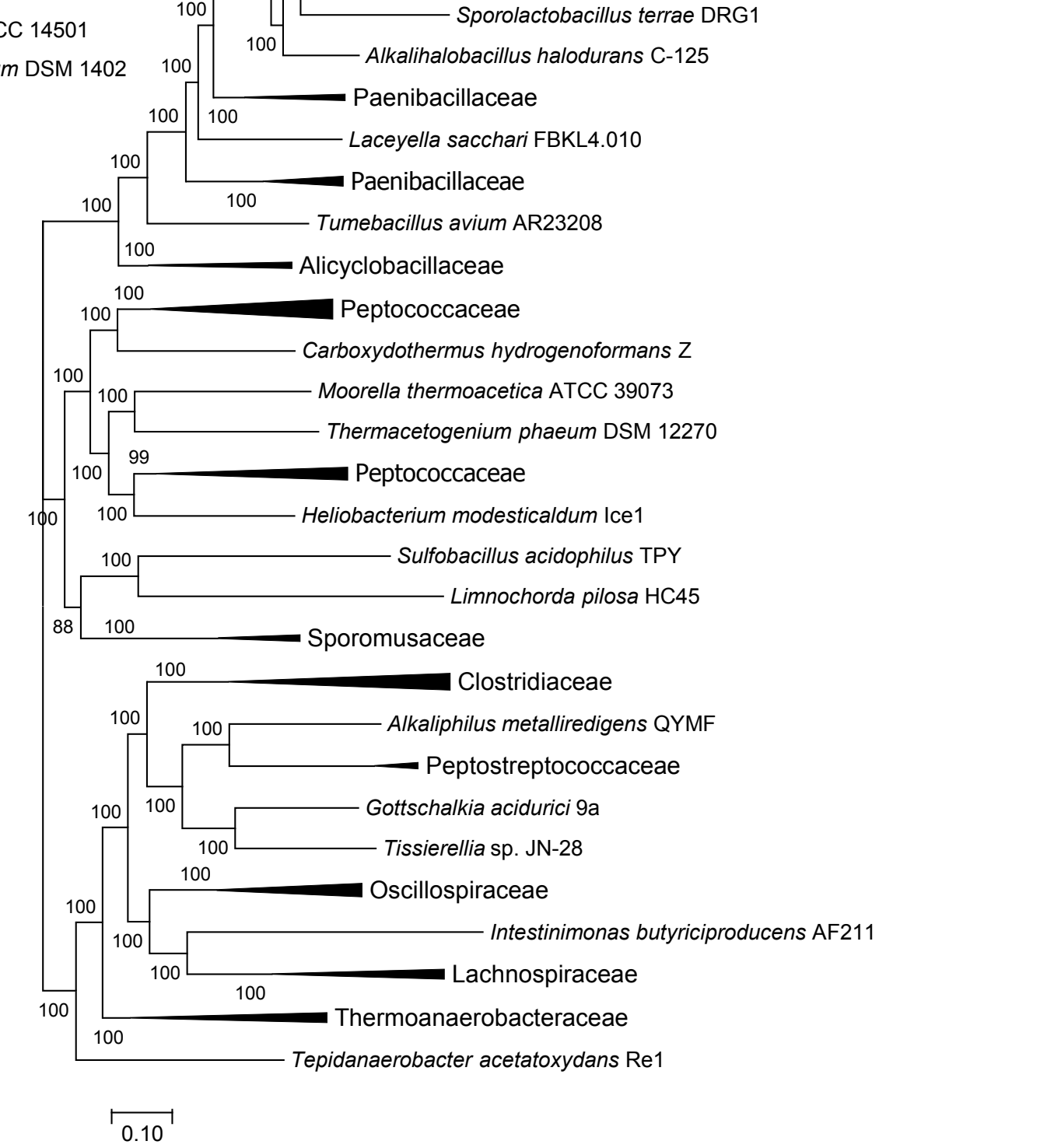

